\title{
Automatic Inference of Determinacy and Mutual Exclu- sion for Logic Programs Using Mode and Type Analyses
}

\author{
Pedro LOPEZ-GARCIA ${ }^{1,2}$, Francisco BUENO ${ }^{3}$ and \\ Manuel HERMENEGILDO ${ }^{1,3}$ \\ ${ }^{1}$ IMDEA Software, Madrid, SPAIN \\ \{pedro.lopez, manuel.hermenegildo\}@imdea.org \\ ${ }^{2}$ Spanish Research Council (CSIC), SPAIN \\ ${ }^{3}$ Technical University of Madrid (UPM), SPAIN \\ \{bueno, herme\}@fi.upm.es
}

\begin{abstract}
We propose an analysis for detecting procedures and goals that are deterministic (i.e., that produce at most one solution at most once), or predicates whose clause tests are mutually exclusive (which implies that at most one of their clauses will succeed) even if they are not deterministic. The analysis takes advantage of the pruning operator in order to improve the detection of mutual exclusion and determinacy. It also supports arithmetic equations and disequations, as well as equations and disequations on terms, for which we give a complete satisfiability testing algorithm, w.r.t. available type information. Information about determinacy can be used for program debugging and optimization, resource consumption and granularity control, abstraction carrying code, etc. We have implemented the analysis and integrated it in the CiaoPP system, which also infers automatically the mode and type information that our analysis takes as input. Experiments performed on this implementation show that the analysis is fairly accurate and efficient.
\end{abstract}

\section{$\S 1$ Introduction}

Knowing that certain predicates are deterministic for a given class of calls has a number of interesting applications such as detecting programming errors, 
performing certain high-level program transformations for improving search efficiency, optimizing low level code generation and parallel execution, and estimating tighter upper bounds on the computational costs of goals and data sizes, which can be used for program debugging, resource consumption and granularity control, abstraction carrying code, etc.

By a predicate being deterministic we mean that it produces at most one solution at most once. It is also interesting to detect predicates whose clauses are mutually exclusive (which implies that at most one of them will succeed) even if they are not deterministic, because they call other predicates that can produce more than one solution (i.e., that are not deterministic). In this paper, we propose a method whereby we can detect procedures and goals that are deterministic, or predicates whose clauses are mutually exclusive. Moreover, we show that, given (upper approximations of) mode and type information, it is feasible to fully automatize our approach, yielding an effective automatic determinacy analysis. The paper is an extended version of our previous proposal, ${ }^{17)}$ which includes more detailed descriptions of the algorithms, more illustrative examples, and more theorems about the termination, correctness or completeness of the algorithms.

There has been much interest on determinacy detection in the literature (see ${ }^{12,14)}$ and its references), using several different forms of determinism. Arguably, one of the first practical determinacy analyses was the one proposed by Sahlin, ${ }^{23)}$ in the context of the Mixtus partial evaluator. This analysis was later reconstructed and semantically justified, using a denotational semantics of Prolog programs with cut, by Mogensen. ${ }^{20)}$ The motivation behind this determinacy analysis was, indeed, to be able to unfold predicates with cuts in their clauses. Therefore, the analysis concentrated on the cut and the control flow of the program: interestingly, the proposal in ${ }^{23)}$ does not take into account predicate arguments. Using a small database of number of possible solutions of built-ins and an analysis of the control structure of the program, estimations of the number of solutions of predicates were performed. The accuracy of this approach has limitations and this is one of the reasons why we explore instead an approach based on the handling of built-ins as tests.

The line of work closest to ours starts with ${ }^{6)}$, in which functional computations are detected and exploited. However, the notion of mutual exclusion in this work (and in our proposal, ${ }^{17}$ ) of which this paper is an extended version, as already mentioned) is not based on constraint satisfaction. This concept is also used in the analysis presented in ${ }^{5}$, where, nonetheless, no algorithms are provided for the detection of mutual exclusion and also the cut is not taken into account. In ${ }^{9)}$, a combined analysis of modes, types, and determinacy is presented, as well as in the more accurate. ${ }^{2)}$ As we will show, our analysis improves on these proposals.

A notion of constraint satisfaction is also present in the approach of ${ }^{14,19}$, which might be considered complementary to ours. Their analyses differ from ours in that they are not goal-oriented and in the mutual exclusion conditions. In particular, the first work ${ }^{19)}$ does not handle the cut, and cannot exploit cer- 
tain program tests that select clauses on execution (e.g., arithmetic tests) which our proposal handles. The second work $^{14)}$ remedies these deficiencies. Still, it concentrates on inferring determinacy conditions, not on checking them. The conditions of ${ }^{14)}$ are richer than ours, since they use success pattern analysis to infer them, based on size relationships between arguments and depth-k abstractions, together with backward analysis. Determinacy conditions are then synthesised in the form of rigidity formulas. For checking them a rigidity analysis is required, to test whether the (propositional) formula holds or not. Instead, we focus on the checking and not on building the conditions. For conditions, we use tests on the instantiation state of arguments which are simply collected from the program text. For the checking, classical mode and type analyses are instrumental. Indeed, our main contribution is a procedure to check satisfiability of the tests which is complete, disregarding how conditions are synthesised.

Several programming systems also make use of determinacy, e.g., Mercury ${ }^{10,24)}$ and HAL. ${ }^{\text {?) }}$ The Mercury and HAL systems allow the programmer to declare that a predicate will produce at most one solution, and attempt to verify this with respect to the Herbrand terms with unification tests. As far as we know, both systems use the same analysis, ${ }^{10)}$ which does not handle disunification tests on the Herbrand domain. This approach also does not handle arithmetic tests, except in the context of the if-then-else construct. As such, it is considerably weaker than the approach described here. Also, our approach does not require any annotations from programmers, since the types and modes on which it is based are inferred. In other words, in addition to proposing concrete algorithms, we also show in this paper that our determinacy analysis can be performed automatically, and is feasible, accurate, and efficient. We do this by integrating it into the Ciao programming system, in particular, into its preprocessor, CiaoPP ${ }^{11)}$ which performs analysis, debugging, verification and optimization tasks, and thus connecting the determinacy analysis with state-ofthe-art type and mode analyses.

\section{$\$ 2$ Preliminaries}

A goal, a class of goals, or a predicate (i.e., all goals for it) are deterministic when they produce at most one solution at most once. When reasoning about determinacy, it is a necessary condition (but not sufficient) that clauses of the predicate be pairwise mutually exclusive, i.e., that only one clause will produce solutions. Additionally, it has to produce only one solution. For reasoning about mutual exclusion, one needs to gather success patterns for each predicate clause, i.e., constraints that the solutions produced by the clause satisfy. Then the basic condition for mutual exclusion is that such success patterns cannot be satisfied simultaneously. This is checked for against available information on the goals being analyzed for determinacy.

We will be using as success patterns tests, which will be unification equations and disequations on terms, and linear equations and disequations on integers or reals. For the checking, we will assume that type information is available, generally as the result of a previous analysis. For concreteness, the determinacy 
analysis we describe is based on regular types, ${ }^{4}$, which are specified by regular term grammars, as explained below, although the concepts should be easily adaptable to other type systems.

\subsection{Regular Types}

A type is a set of (Herbrand) terms, and can be defined by using a number of different representations, such as type terms and regular term grammars as in ${ }^{4)}$, or type graphs as in ${ }^{13)}$ ), or simply predicates as in the Ciao system. ${ }^{3}$ ) We will use the formalism of ${ }^{4}$, and summarize below the relevant concepts.

A type symbol is an abstraction of a set of Herbrand terms (i.e., of a type). We use the Greek letter $\alpha$ for referring to type symbols in general (with subscripts if necessary). The $\gamma$ function maps each type symbol to the set of Herbrand terms that it represents. Given a type symbol $\alpha$, the set of terms (i.e., the type) represented by it is denoted as $\gamma(\alpha)$. To enhance readability, we abuse notation and use $\alpha$ instead of $\gamma(\alpha)$ when no ambiguity is possible.

We assume the existence of an infinite set of type symbols, which is disjoint with the sets of constant symbols, function symbols, and variables. There are two special type symbols: $\mu$, that represents the type of the entire Herbrand universe; and $\phi$, that represents the empty type (i.e., $\gamma(\phi)=\emptyset$ ). There is a distinguished non-empty finite subset of the set of type symbols called the set of base type symbols, which represent base types. For any base type $\alpha$, we assume that $\gamma(\alpha)$ is infinite and there are effective tests for membership of a given Herbrand term in $\gamma(\alpha)$.

\section{Example 2.1}

Examples of base type symbols that we use in our determinacy analysis are: int, such that the base type $\gamma($ int $)$ is the set of all constant symbols that represent integer numbers; and atm, such that the base type $\gamma(\mathrm{atm})$ is the set of all constant symbols that do not represent numbers.

A type term is either a constant symbol, a variable, a type symbol, or a term $f\left(\omega_{1}, \ldots, \omega_{n}\right)$, where $f$ is an $n$-ary function symbol, and each $\omega_{i}$ is a type term. Note that all type symbols are type terms, however, the converse is not true. A pure type term is one which does not contain variables. A Herbrand term is a type term which does not contain type symbols (it can contain variables).

A type rule is an expression of the form $\alpha \rightarrow \Upsilon$, where $\alpha$ is a type symbol, and $\Upsilon$ is a set of pure type terms. We denote sets of type rules, that is, regular term grammars, by the letter $T$ (as in ${ }^{4)}$ ). A (non-base) type symbol $\alpha$ is defined in, or by, a set of type rules $T$ if there exists a type rule $(\alpha \rightarrow \Upsilon) \in T$. A pure type term $\omega$ is defined by a set of type rules $T$ if each type symbol in $\omega$ is either $\mu, \phi$, a base type symbol, or a (non-base) type symbol defined in $T$. We assume that, for each type rule $(\alpha \rightarrow \Upsilon) \in T$, each element (i.e., pure type term) of $\Upsilon$ is defined in $T$, and that each type symbol defined in $T$ has exactly one defining type rule in $T$. Moreover, we will also assume that every type rule is deterministic, i.e., every element of $\Upsilon$ is a base type symbol or a pure type term of the form $f\left(\alpha_{1}, \ldots, \alpha_{n}\right), n \geq 0$, and there is no pair of pure type terms 
$\omega_{1}, \omega_{2} \in \Upsilon$, such that $\omega_{1} \neq \omega_{2}, \omega_{1}=f\left(\omega_{1}^{1}, \ldots, \omega_{n}^{1}\right)$, and $\omega_{2}=f\left(\omega_{1}^{2}, \ldots, \omega_{n}^{2}\right)$ (i.e., there is no pair of pure type terms in $\Upsilon$ with the same principal functor). The class of types that can be described by deterministic type rules is the same as the class of tuple-distributive regular types. ${ }^{4)}$ Additional background on type-related issues may be found in ${ }^{4,13)}$.

\section{Example 2.2}

The type rule list $\rightarrow\{[],[\mu \mid$ list $]\}$ defines the type symbol list, that denotes the set of all lists. The type rule intlist $\rightarrow\{[],[$ int $\mid$ intlist $]\}$ defines the type symbol intlist, that denotes the set of all lists of integer numbers.

It is also possible to provide polymorphism in our setting. Since we use types for describing instantiation patterns, a polymorphic type such as, e.g., list $(\alpha) \rightarrow\{[],[\alpha \mid$ list $(\alpha)]\}$ is useful only in the description of the list structure, but not of the elements. Thus, the instance type list $(\mu)$ (i.e., list) serves the same purposes. Instances of polymorphic types are thus "computed away" (so that, e.g., list (int) yields intlist) and our approach handles them in this way.

Given a predicate $q$ in a program $P$, type $[q]$ denotes a tuple of pure type terms representing the types of the arguments of predicate $q$. In the interest of simplicity, we abuse terminology and say that type $[q]$ is the type of predicate $q$. In this paper, we are concerned exclusively with calling types for predicates -in other words, when we say "a predicate $q$ in a program $P$ has type type $[q]$ ", we mean that in any execution of the program $P$ starting from some class of queries of interest, whenever there is a call $q(\bar{t})$ to the predicate $q$, the argument tuple $\bar{t}$ in the call will be an element of the set denoted by type $[q]$.

\section{Definition 2.1 (type assignment)}

Given a (finite) tuple of variables $\bar{x}=\left(x_{1}, \ldots, x_{n}\right)$, a type assignment $\rho$ over $\bar{x}$ maps each variable $x_{i}$ for $1 \leq i \leq n$ to a (nonempty) pure type term $\omega_{i}$, i.e., $\rho\left(x_{i}\right)=\omega_{i}$. We write the type assignment $\rho$ as $\bar{x}: \bar{\omega}$, where $\bar{\omega}$ is the tuple of pure type terms $\left(\omega_{1}, \ldots, \omega_{n}\right)$. However, we sometimes abuse of notation and write $\rho$ as $\left(x_{1}: \omega_{1}, \ldots, x_{n}: \omega_{n}\right)$.

\subsection{Tests (and Modes)}

We define a test to be either a primitive test, or a conjunction $\tau_{1} \wedge \tau_{2}$, or a disjunction $\tau_{1} \vee \tau_{2}$, or a negation $\neg \tau_{1}$, where $\tau_{1}$ and $\tau_{2}$ are tests. A primitive test is a positive literal whose predicate symbol is a built-in such as the unification or some arithmetic built-in predicate $(<,>, \leq, \geq, \neq$, etc.) which acts as a "test" (note that with our assumptions of having available both mode and type information for each variable in a program, it is straightforward to identify primitive tests in a program). Primitive tests which are true of the successes of a given clause are gathered together to form the test of that clause. For concreteness, in our experiments (Section 5), we will gather for each clause the primitive tests occurring in the program text of that clause. One could use more sophisticated approaches, such as backwards analysis with a depth-k abstraction. ${ }^{12)}$ Our approach remains valid regardless of the means used to build the tests. 
For example, if term structure information is available, it will be used in the algorithms below as if it appeared in the program text.

Because of limitations of state-of-the-art technology in type analysis, primitive tests have to be carefully selected. Actual, working type analyses infer types which denote sets of terms that are closed under substitution. On the contrary, our algorithms will be based on types which denote sets of ground terms. The gap between these two classes of types is covered with the use of modes.

In practice, the difference amounts to the interpretation of the universal type symbol $\mu$. In the ground interpretation, $\mu$ denotes the set of all ground terms. Otherwise, $\mu$ (i.e., the classical top in type analyses) also denotes terms which may contain variables. This issue is important in deciding whether certain (unification) literals can act as tests or not and, therefore, whether they can be used in mutual exclusion conditions or not. For example, consider two tests $\mathrm{X}=[\mathrm{a}]$ and $\mathrm{X}=[\mathrm{b}]$ for different clauses. Assume we are analyzing goals which satisfy the type assignment (X) : ( $\alpha)$ with type rule $\alpha \rightarrow\{[\mu]\}$. In the ground interpretation, the two tests are mutually exclusive, but they are not in the other interpretation (since the head of the list constructor in $\mathrm{X}$ might be a free variable). Mode information is then essential in distinguishing such cases.

In our experiments, we will use groundness and freeness information obtained from a sharing analysis to establish the modes. This information is used to classify primitive tests, and only those regarded as input tests will be considered when building tests for clauses. Input tests perform a comparison of (numerical) values or a matching of terms, rather than a proper unification. Given mode and type information on the program, it is straightforward to identify them.

\section{Example 2.3}

Consider the literal $\mathrm{X}$ is $\mathrm{Y}+1$ appearing in the body of a clause. If the available mode and type information asserts that, just before calling this literal, variables $\mathrm{X}$ and $\mathrm{Y}$ are bound to integer numbers, then the literal is considered a primitive (arithmetic) input test. However, if the mode and type information asserts that $\mathrm{X}$ is an unbound variable and $\mathrm{Y}$ is bound to an integer, then the literal acts as an assignment and thus is not considered a test. If there is a body literal of the form $\mathrm{X}=\mathrm{Y}$ and the information asserts that variables $\mathrm{X}$ and $\mathrm{Y}$ will be bound to ground terms upon call, then the literal is considered to be a primitive (unification) input test. If the information asserts that any of the variables $\mathrm{X}$ or $\mathrm{Y}$ are free, then the literal is not considered a test.

Where necessary to emphasize the input test in a clause, we will write the clause in "guarded" form. As an example, consider a predicate that is called as $\operatorname{abs}(X, Y)$, where $X$ is bound to an integer and $Y$ is a free variable, to obtain the absolute value of $X$. Its definition will be written as:

$$
\begin{aligned}
& \operatorname{abs}(X, Y):-X \geq 0 \rrbracket Y=X . \\
& \operatorname{abs}(X, Y):-X<0 \rrbracket Y \text { is }-X .
\end{aligned}
$$

Obviously, for any particular call in the class above, only one of the tests $\mathrm{X} \geq 0$ or $\mathrm{X}<0$ will succeed (i.e., the tests are mutually exclusive). 
Note that the distinction between tests and input tests is due only to limitations in the technology used in our experiments. In fact, we will be using the word test throughout the rest of the paper when talking about mutual exclusion conditions. The following definition characterizes tests and will be instrumental in the formal results:

\section{Definition 2.2 (solutions of a test)}

Given a test $\tau(\bar{x})$, Sols $(\tau(\bar{x}))$ is the set of all tuples of ground terms $\bar{e}$ which are instances of $\bar{x}$ such that $\bar{x}=\bar{e} \wedge \tau(\bar{x})$ is satisfiable (i.e., test $\tau(\bar{e})$ succeeds).

\subsection{Mutual Exclusion}

Fundamental to our approach to detecting determinacy is the notion of tests being "exclusive" w.r.t. a type assignment:

\section{Definition 2.3}

Two tests $\tau_{1}(\bar{x})$ and $\tau_{2}(\bar{x})$ are exclusive w.r.t. a type assignment $\bar{x}: \bar{\omega}$, if for every $\bar{t} \in \gamma(\bar{\omega}), \bar{x}=\bar{t} \wedge \tau_{1}(\bar{x}) \wedge \tau_{2}(\bar{x})$ is unsatisfiable.

\section{Definition 2.4 (mutual exclusion)}

Let $C_{1}, \ldots, C_{n}, n>0$, be a sequence of clauses, with input tests $\tau_{1}(\bar{x}), \ldots, \tau_{n}(\bar{x})$ respectively. Let $\rho$ be a type assignment. We say that $C_{1}, \ldots, C_{n}$ is mutually exclusive w.r.t. $\rho$ if either, $n=1$, or, for every pair of clauses $C_{i}$ and $C_{j}$, $1 \leq i, j \leq n, i \neq j, \tau_{i}(\bar{x})$ and $\tau_{j}(\bar{x})$ are exclusive w.r.t. $\rho$.

Consider a predicate $p$ defined by $n$ clauses $C_{1}, \ldots, C_{n}$, with input tests $\tau_{1}(\bar{x}), \ldots, \tau_{n}(\bar{x})$ respectively. Let predicate $p$ have type type $[p]$ : in the interest of simplicity, we sometimes say that predicate $p$ is mutually exclusive w.r.t. the type type $[p]$ (or simply that predicate $p$ is mutually exclusive) if the sequence of clauses $C_{1}, \ldots, C_{n}$ defining $p$ is mutually exclusive w.r.t. the type assignment $\bar{x}$ : type $[p]$. Given a call $c$ to predicate $p$ in the body of a clause, we also say that $c$ is mutually exclusive if $p$ is. Note that if the predicate $p$ is mutually exclusive, then at most one of its clauses will succeed for any call $p(\bar{t})$, with $\bar{t} \in \gamma($ type $[p])$.

\section{$\S 3$ Determinacy Analysis}

In this section, we explain our algorithm for detecting predicates and calls that are deterministic. Before introducing our algorithm, we give some instrumental definitions. We define the "calls" relation between predicates in a program as follows: $p$ calls $q$, written $p \leadsto q$, if and only if a literal with predicate symbol $q$ appears in the body of a clause defining $p$. Let $\sim^{\star}$ denote the reflexive transitive closure of $\leadsto$. The following result shows the importance of mutual exclusion information for detecting determinacy.

\section{Theorem 3.1}

A predicate $p$ in the program is deterministic if, for each predicate $q$ such that $p \leadsto{ }^{\star} q, q$ is mutually exclusive. 


\section{Proof}

Assume that $p$ is not deterministic, i.e., there is a goal $p(\bar{t})$, with $\bar{t} \in$ type $[p]$, which is not deterministic. It is a straightforward induction on the number of resolution steps to show that there is a $q$ such that $p \sim^{\star} q$ and $q$ is not mutually exclusive.

Our algorithm for detecting determinacy consists of first determining which predicates are mutually exclusive (which is in fact the convoluted part, and is explained in detail in Section 4). Then, inferring determinacy is straightforward: from Theorem 3.1, analysis of determinacy reduces to the determination of reachability in the call graph of the program. In other words, a predicate $p$ is deterministic if there is no path in the call graph of the program from $p$ to any predicate $q$ that is not mutually exclusive. It is straightforward to propagate this reachability information in a single traversal of the call graph in reverse topological order. The idea is illustrated by the following example.

\section{Example 3.1}

Consider the classical quicksort program with a main calling mode in which the first argument is ground and the second one is free. Figure 1 shows the guarded version of the program for this mode. Assume calling type (intlist, -) for qs $/ 2$. The calling types for part/4 and app/3 are (intlist, int, -, -) and (intlist, intlist, -) respectively. Since determinacy analysis traverses the call graph in reverse topological order, it considers first predicates part $/ 4$ and app $/ 3$.

The input tests for the clauses of part (L,C, Left, Right) are $\tau_{1}^{\text {part }}(\mathrm{L}, \mathrm{C})$ $\equiv \mathrm{L}=[], \tau_{2}^{\text {part }}(\mathrm{L}, \mathrm{C}) \equiv \mathrm{L}=[\mathrm{E} \mid \mathrm{R}] \wedge \mathrm{E}<\mathrm{C}$ and $\tau_{3}^{\text {part }}(\mathrm{L}, \mathrm{C}) \equiv \mathrm{L}=[\mathrm{E} \mid \mathrm{R}] \wedge \mathrm{E}>=\mathrm{C}$. According to the calling type, the analysis uses the type assignment $\rho^{\text {part }} \equiv$ $(\mathrm{L}, \mathrm{C})$ : (intlist, int), and infers that $\tau_{i}^{\text {part }}(\mathrm{L}, \mathrm{C}), i=1,2,3$ are mutually exclusive w.r.t. $\rho^{\text {part }}$ (we will explain the details in Section 4). It means that at most one of these tests will succeed. Thus, clauses of part/4 are mutually exclusive. It follows that calls to part $/ 4$ which satisfy the calling types are deterministic.

Similarly, the input tests for the sequence of clauses of app (L1, L2, L3) are $\tau_{1}^{a p p}(\mathrm{~L} 1, \mathrm{~L} 2) \equiv \mathrm{L} 1=[]$ and $\tau_{2}^{a p p}(\mathrm{~L} 1, \mathrm{~L} 2) \equiv \mathrm{L} 1=[\mathrm{X} \mid \mathrm{Xs}]$. The type assignment

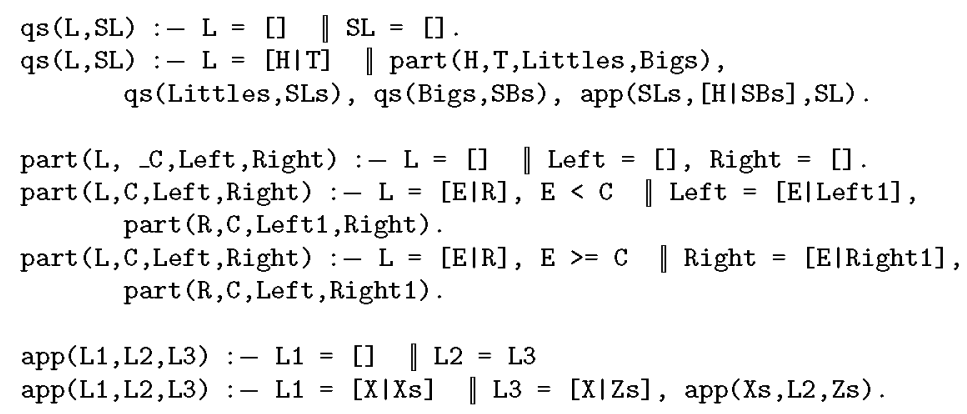

Fig. 1 A Quicksort Program 
$\rho^{a p p}$ corresponding to the calling types for app/3 is ( $\left.11, \mathrm{~L} 2\right)$ : (intlist, intlist). The analysis infers that $\tau_{1}^{a p p}(\mathrm{~L} 1, \mathrm{~L} 2)$ and $\tau_{2}^{a p p}(\mathrm{~L} 1, \mathrm{~L} 2)$ are exclusive w.r.t. the type assignment $\rho^{a p p}$. Thus, it follows that calls to app $/ 3$ which satisfy the calling types are also deterministic.

Finally, the input tests for the sequence of clauses of qs (L, SL) are $\tau_{1}^{q s}(\mathrm{~L}) \equiv$ $\mathrm{L}=[]$ and $\tau_{2}^{q s}(\mathrm{~L}) \equiv \mathrm{L}=[\mathrm{H} \mid \mathrm{T}]$. The type assignment $\rho^{q s}$ corresponding to the calling types is (L): (intlist). We have that $\tau_{1}^{q s}(\mathrm{~L})$ and $\tau_{2}^{q s}(\mathrm{~L}$ ) are exclusive w.r.t. type assignment $\rho^{q s}$. Thus, clauses of qs $/ 2$ are mutually exclusive. Moreover, since the calls to the predicates part $/ 4$ and app $/ 3$ in the body of the clauses defining qs $/ 2$ have been proved to be deterministic, it follows that calls to qs $/ 2$ with the first argument bound to a list of integers are deterministic.

\subsection{Improving Determinacy Analysis using Cut}

The presence of pruning operators in program clauses can be exploited to improve the overall process of detecting deterministic predicates. Besides helping the detection of mutual exclusion of clauses (as we will see below in Section 4.4), it can also improve the propagation algorithm given above. Assume that we want to infer whether a predicate $p$ is deterministic. Consider any clause defining $p$ in which one or more cuts appear, and any body literals that appear to the left of the rightmost cut in that clause. Those literals are not required to be deterministic. In other words, in Theorem 3.1, we can use a restricted definition $\left(\sim_{r}\right)$ of the "call" relation $(\leadsto)$ between predicates in a program, defined as follows: $p \sim_{r} q$, if and only if a literal with predicate symbol $q$ appears in the body of a clause defining $p$, and there is no cut to the right of this literal in the clause. Similarly, $\sim_{r}^{*}$ denotes the reflexive transitive closure of $\sim_{r}$.

\section{$\S 4$ Checking Mutual Exclusion}

Our approach to the problem of determining whether a set of tests $\tau_{i}(\bar{x})$ for $i=1, \ldots, n$ are mutually exclusive w.r.t. a type assignment $\bar{x}: \bar{\omega}$, consists of reducing the problem to subproblems, each subproblem involving tests of the same type, i.e., defining a particular constraint system. Each subproblem is solved by applying an algorithm that is specific to the corresponding constraint system that checks mutual exclusion. In this paper, we consider two commonly encountered constraint systems: Herbrand terms with unification and disunification tests, on variables with tuple-distributive regular types ${ }^{4)}$ (see Section 2.1) and linear arithmetic tests on integer variables.

\section{Example 4.1}

Consider the predicate part/4 taken from the quicksort program shown in Fig.1. For the sequence of clauses of part ( $\mathrm{L}, \mathrm{C}$, Left, Right), we have three input tests $\tau_{i}(\bar{x}), i=1,2,3$, where $\bar{x} \equiv(\mathrm{L}, \mathrm{C})$ in this case. As commented in Example 3.1, the input tests are (omitting $\bar{x}$ and the superscript part for simplicity): $\tau_{1} \equiv \mathrm{L}=[]$, $\tau_{2} \equiv \mathrm{L}=[\mathrm{E} \mid \mathrm{R}] \wedge \mathrm{E}<\mathrm{C}$ and $\tau_{3} \equiv \mathrm{L}=[\mathrm{E} \mid \mathrm{R}] \wedge \mathrm{E}>=\mathrm{C}$. We will separate Herbrand tests from arithmetic tests and write $\tau_{1}$ as $\tau_{1}^{H} \wedge \tau_{1}^{A}$, where $\tau_{1}^{H} \equiv \mathrm{L}=[]$ and 
$\tau_{1}^{A} \equiv$ true. Similarly, $\tau_{2}^{H} \equiv \mathrm{L}=[\mathrm{E} \mid \mathrm{R}]$ and $\tau_{2}^{A} \equiv \mathrm{E}<\mathrm{C}$, and $\tau_{3}^{H} \equiv \mathrm{L}=[\mathrm{E} \mid \mathrm{R}]$ and $\tau_{3}^{A} \equiv \mathrm{E}>=\mathrm{C}$.

We have to check that the tests $\tau_{i}(\bar{x}), i=1,2,3$, are mutually exclusive w.r.t. the type assignment $\rho \equiv(\mathrm{L}, \mathrm{C})$ : (intlist, int). This problem is reduced to two subproblems: a) Checking that the tests $\mathrm{L}=[]$ and $\mathrm{L}=[\mathrm{E} \mid \mathrm{R}]$ are exclusive w.r.t. $\rho$, which prove that $\tau_{1}$ and $\tau_{2}$ (as well as $\tau_{1}$ and $\tau_{3}$ ) are exclusive (since the Herbrand parts of the tests are exclusive), and b) Checking that the tests $\mathrm{E}<\mathrm{C}$ and $\mathrm{E}>=\mathrm{C}$ are exclusive w.r.t. the type assignment ( $\mathrm{C}, \mathrm{E})$ : (int, int), which prove that $\tau_{2}$ and $\tau_{3}$ are exclusive. In this second subproblem, the Herbrand parts of the tests are not exclusive (in fact, both of them are the same test, $\mathrm{L}=[\mathrm{E} \mid \mathrm{R}]$ ), and hence, it is necessary to check the exclusion of the arithmetic parts.

\subsection{Checking Mutual Exclusion in the Herbrand Domain}

We present a decision procedure for checking mutual exclusion of tests that is inspired by a result, due to Kunen ${ }^{15}{ }^{5}$ that establishes that the emptiness problem is decidable for Boolean combinations of (notations for) certain "basic" subsets of the Herbrand Universe of a program. It also uses straightforward adaptations of some operations described by Dart and Zobel. ${ }^{4)}$ The reason the mutual exclusion checking algorithm for Herbrand is as convoluted as it is, is that we want a complete algorithm for unification and disunification tests. It is possible to make it more clear if we are interested in unification tests only.

Before describing the algorithm, we introduce some definitions and notation. We denote the Herbrand Universe (i.e., the set of all ground terms) as $\mathcal{H}$, and the set of $n$-tuples of elements of $\mathcal{H}$ as $\mathcal{H}^{n}$. We use the notions (to be defined in the following) of type-annotated term, and in general elementary set, as representations which denote some subsets of $\mathcal{H}^{n}$ (for some $n \geq 1$ ). These subsets can be, for example, the set of $n$-tuples for which a test succeeds, or a calling type for a predicate $p$ (i.e., the set denoted by type $[p]$ ). Given a representation $S$ (elementary set or type-annotated term), the denotation of $S, \operatorname{Den}(S)$ refers to the subset of $\mathcal{H}^{n}$ denoted by $S$.

\section{Definition 4.1 (type-annotated term)}

A type-annotated term $\delta$ is a pair $\left(\bar{t}_{\delta}, \rho_{\delta}\right)$, where $\bar{t}_{\delta}$ is a tuple of terms, and $\rho_{\delta}$ is a type assignment.

We will represent type-annotated terms with the symbol $\delta$ possibly subscripted. Given a type-annotated term $\delta=\left(\bar{t}_{\delta}, \rho_{\delta}\right)$, the denotation of $\delta, \operatorname{Den}(\delta)$ is the set of all the ground terms $\bar{t}_{\delta} \theta$, where $\theta$ is some substitution, such that $x \theta \in \gamma\left(\rho_{\delta}(x)\right)$ for each variable in $\bar{t}_{\delta}$. In other words, $\operatorname{Den}(\delta)$ is the set of all the ground instances of $\bar{t}_{\delta}$ resulting from replacing the variables in $\bar{t}_{\delta}$ by a term belonging to the type assigned to those variables by $\rho_{\delta}$.

\section{Example 4.2}

We define some examples of type-annotated terms $\delta_{1}, \delta_{2}$, and $\delta_{3}$ as follows: $\delta_{1}=$ $\left((x, y),(x, y):\left(\alpha_{1}, \alpha_{2}\right)\right)$, where $\alpha_{1} \rightarrow\{f(\mu)\}$ and $\alpha_{2} \rightarrow\{g(\mu), h(\mu)\} ; \delta_{2}$ is the 
type-annotated term $\left(\bar{t}_{2}, \rho_{2}\right)$ such that $\bar{t}_{2} \equiv(f(z), w)$ and $\rho_{2} \equiv(z, w):\left(\mu, \alpha_{2}\right)$ (note that $\delta_{1}$ and $\delta_{2}$ denote the same subset of $\mathcal{H}^{2}$, i.e., $\left.\operatorname{Den}\left(\delta_{1}\right)=\operatorname{Den}\left(\delta_{2}\right)\right) ; \delta_{3}$ is the type-annotated term $\left(\bar{t}_{3}, \rho_{3}\right)$ with $\bar{t}_{3} \equiv\left(f\left(v_{1}\right), g\left(v_{2}\right), v_{3}, v_{4}, f(a), f\left(v_{5}\right), v_{6}\right)$ and $\rho_{3} \equiv\left(v_{1}, v_{2}, v_{3}, v_{4}, v_{5}, v_{6}\right):\left(\mu\right.$, list, $\alpha_{2}, \alpha_{3}, \alpha_{3}$, list $)$, where $\alpha_{3} \rightarrow\{a, b\}$ and list $\rightarrow\{[],[\mu \mid$ list $]\}$.

Given a type-annotated term $(\bar{t}, \rho)$, the tuple of terms $\bar{t}$ can be regarded as a Herbrand term (i.e., a type-symbol-free type term) and $\rho$ can be considered to be a type substitution, ${ }^{* 1}$ so that, if we apply this type substitution to $\bar{t}$, we get a pure type term (a variable-free type term). This is useful for defining the "intersection" and "inclusion" operations over type-annotated terms (that we define later), using the algorithms described by Dart and Zobel ${ }^{4)}$ for performing these operations over pure type terms. When we have a type-annotated term $(\bar{t}, \rho)$ such that $\rho(x)=\mu$ for each variable $x$ in $\bar{t}$, we omit the type assignment $\rho$ for brevity and use the tuple of terms $\bar{t}$. Thus, a tuple of terms $\bar{t}$ with no associated type assignment can be regarded as a type-annotated term which denotes the set of all ground instances of $\bar{t}$.

\section{Definition 4.2 (elementary set)}

An elementary set is defined as follows:

- $\Lambda$ is an elementary set.

- A type-annotated term $(\bar{t}, \rho)$ is an elementary set.

- If $A$ and $B$ are elementary sets, then $A \otimes B, A \oplus B$ and $\operatorname{comp}(A)$ are elementary sets.

Since we have already defined the denotation of type-annotated terms, we define now the denotation of the rest of elementary sets.

- $\operatorname{Den}(\Lambda)=\emptyset$ (the empty set).

- If $A$ and $B$ are elementary sets, then $\operatorname{Den}(A \otimes B)=\operatorname{Den}(A) \cap \operatorname{Den}(B)$, $\operatorname{Den}(A \oplus B)=\operatorname{Den}(A) \cup \operatorname{Den}(B)$ and $\operatorname{Den}(\operatorname{comp}(A))=\mathcal{H}^{n} \backslash \operatorname{Den}(A)$.

We also define the following relations between elementary sets:

- $A \sqsubseteq B$ iff $\operatorname{Den}(A) \subseteq \operatorname{Den}(B)$.

- $A \sqsubset B$ iff $\operatorname{Den}(A) \subset \operatorname{Den}(B)$.

- $A \simeq B$ iff $\operatorname{Den}(A)=\operatorname{Den}(B)$.

We define below two particular classes of elementary sets, namely, cobasic sets and minsets, which are suitable representations of tests for the algorithms that we present in this paper. A test $\tau(\bar{x})$ that is a conjunction of unification and disunification tests is represented as a minset that denotes the set of ground instances of $\bar{x}$ (i.e., subsets of $\mathcal{H}^{n}$, assuming that $\bar{x}$ is a $n$-tuple) for which the test succeeds. A disunification test is represented by a cobasic set (which denotes the complementary set of a subset of $\mathcal{H}^{n}$ ).

*1 A type substitution is similar to a substitution that maps variables to type terms. A detailed definition of type substitutions is given in ${ }^{4)}$. 


\section{Definition 4.3 (cobasic set)}

A cobasic set is an elementary set of the form comp $(\bar{t})$, where $\bar{t}$ is a tuple of terms.

\section{Definition 4.4 (minset)}

A minset is either $\Lambda$ or an elementary set of the form $\bar{t}_{0} \otimes \operatorname{comp}\left(\bar{t}_{1}\right) \otimes \cdots \otimes$ $\operatorname{comp}\left(\bar{t}_{n}\right)$, for some $n \geq 0$, where:

- $\bar{t}_{0}$ is a tuple of terms,

- $\operatorname{comp}\left(\bar{t}_{1}\right), \ldots, \operatorname{comp}\left(\bar{t}_{n}\right)$ are cobasic sets,

- for all $i, 1 \leq i \leq n, \bar{t}_{i} \sqsubset \bar{t}_{0}$ (which implies that $\bar{t}_{i}=\bar{t}_{0} \theta_{i}$ for some substitution $\theta_{i}$ ), and

- for all $i, j$ such that $1 \leq i, j \leq n$ and $i \neq j$, it holds that $\bar{t}_{i} \nsubseteq \bar{t}_{j}$.

For brevity, we write a minset of the form $\bar{t}_{0} \otimes \operatorname{comp}\left(\bar{t}_{1}\right) \otimes \cdots \otimes \operatorname{comp}\left(\bar{t}_{n}\right)$ as $\bar{t}_{0} \otimes \mathcal{C}$, where $\mathcal{C}=\left\{\operatorname{comp}\left(\bar{t}_{1}\right), \ldots, \operatorname{comp}\left(\bar{t}_{n}\right)\right\}$. The minset representation of a test is given by the test2minset function defined below.

\section{Definition 4.5 (exact representation of a test)}

A minset $\beta$ is an exact representation of a test $\tau(\bar{x})$ if $\operatorname{Den}(\beta)=\operatorname{Sols}(\tau(\bar{x}))$. That is, for any tuple of ground terms $\bar{e}$, it holds that $\bar{e} \in \operatorname{Den}(\beta)$ if and only if $\bar{x}=\bar{e} \wedge \tau(\bar{x})$ is satisfiable (i.e., the test $\tau(\bar{e})$ succeeds).

\section{Definition 4.6 (test2minset function)}

We define the test2minset $(\tau(\bar{x}))$ function which takes a test $\tau(\bar{x})$ and returns a minset $\beta$ which is an exact representation of $\tau(\bar{x})$. We assume without lost of generality that $\tau(\bar{x})$ is a conjunction of unification and disunification tests and is of the form $\mathcal{E} \wedge \mathcal{D}_{1} \wedge \cdots \wedge \mathcal{D}_{n}$, where $\mathcal{E}$ is the conjunction of all unification tests of $\tau(\bar{x})$ (i.e., a system of equations) and each $\mathcal{D}_{i}$ a disunification test (i.e., a disequation). The returned value $\beta$ is computed as follows:

1. Let $\theta_{0}$ be the substitution associated with the solved form of $\mathcal{E}$ (this can be computed by using the techniques of Lassez et al. ${ }^{16)}$ ).

2. If $\theta_{0}$ does not exist, then make $\beta=\Lambda$.

3. Otherwise, let $\theta_{i}$, for $1 \leq i \leq n$, be the substitution associated with the solved form of $\mathcal{E} \wedge \mathcal{N}_{i}$, where $\mathcal{N}_{i}$ is the negation of $\mathcal{D}_{i}$.

4. Let $\beta^{\prime}=\bar{t}_{0} \otimes \operatorname{comp}\left(\bar{t}_{1}\right) \otimes \cdots \otimes \operatorname{comp}\left(\bar{t}_{n}\right)$, where $\bar{t}_{i}=\bar{x} \theta_{i}$, if $\theta_{i}$ exists, for $0 \leq i \leq n$ (if $\theta_{i}$ does not exist, then $\operatorname{comp}\left(\bar{t}_{i}\right)$ does not appear in the definition of $\beta^{\prime}$ ).

5. If $\bar{t}_{0} \sqsubseteq \bar{t}_{i}$, for some cobasic set $\operatorname{comp}\left(\bar{t}_{i}\right)$, then make $\beta=\Lambda$.

6. Otherwise, perform a simplification step on $\beta^{\prime}$ by removing all cobasic sets $\operatorname{comp}\left(\bar{t}_{i}\right)$ for which there is a cobasic set $\operatorname{comp}\left(\bar{t}_{j}\right), 1 \leq i, j \leq n$ and $i \neq j$, such that $\bar{t}_{i} \sqsubseteq \bar{t}_{j}$. Make $\beta$ be the resulting minset.

\section{Theorem 4.1}

Let $\tau(\bar{x})$ be a conjunction of unification and disunification tests, and $\beta=$ test2minset $(\tau(\bar{x}))$. Then $\beta$ is an exact representation of $\tau(\bar{x})$. 


\section{Proof}

- Since we use the techniques of Lassez et al. ${ }^{16)}$ ) for computing solved forms of systems of equations over Herbrand terms, it follows that if $\theta_{0}$ does not exist (step 2), then $\mathcal{E}$ is unsatisfiable and hence $\tau(\bar{x})$ also is, thus $\beta=\Lambda$ is an exact representation of $\tau(\bar{x})$.

- For the same reason, if $\theta_{0}$ exists (step 3), then it is a most general unifier, and thus $\bar{t}_{0}$ is an exact representation of $\mathcal{E}$. We can prove it because for any tuple of ground terms $\bar{e}$, it holds that if $\bar{e} \in \operatorname{Den}\left(\bar{t}_{0}\right)$ then $\bar{e}=\bar{t}_{0} \theta_{e}$ for some ground substitution $\theta_{e}$. Since $\bar{t}_{0}=\bar{x} \theta_{0}$, we have that $\bar{e}=\bar{x} \theta_{0} \theta_{e}$. Let $\theta_{e}^{\prime}=\theta_{e} \circ \theta_{0}$, i.e., $\bar{e}=\bar{x} \theta_{e}^{\prime}$. By definition, $\theta_{0}$ is more general than $\theta_{e}^{\prime}$, and thus $\bar{x}=\bar{e} \wedge \mathcal{E}$ is satisfiable. Conversely, if $\bar{x}=\bar{e} \wedge \mathcal{E}$ is satisfiable then $\bar{e}=\bar{x} \theta_{e}^{\prime}$ for some ground substitution $\theta_{e}^{\prime}$ which is more specific than $\theta_{0}$, i.e., $\theta_{e}^{\prime}=\theta_{e} \circ \theta_{0}$, thus $\bar{e} \in \operatorname{Den}\left(\bar{t}_{0}\right)$.

- In step 4 , we have that $\operatorname{Den}\left(\beta^{\prime}\right)=\operatorname{Den}\left(\bar{t}_{0} \otimes \operatorname{comp}\left(\bar{t}_{1}\right) \otimes \cdots \otimes \operatorname{comp}\left(\bar{t}_{n}\right)\right)=$ $\operatorname{Den}\left(\left(\bar{t}_{0} \otimes \operatorname{comp}\left(\bar{t}_{1}\right)\right) \otimes \cdots \otimes\left(\bar{t}_{0} \otimes \operatorname{comp}\left(\bar{t}_{n}\right)\right)\right)=\operatorname{Den}\left(\bar{t}_{0} \otimes \operatorname{comp}\left(\bar{t}_{1}\right)\right) \cap \cdots \cap$ $\left.\operatorname{Den}\left(\bar{t}_{0} \otimes \operatorname{comp}\left(\bar{t}_{n}\right)\right)=\operatorname{Sols}\left(\mathcal{E} \wedge \mathcal{D}_{1}\right) \cap \cdots \cap \operatorname{Sols}\left(\mathcal{E} \wedge \mathcal{D}_{n}\right)\right)=\operatorname{Sols}\left(\mathcal{E} \wedge \mathcal{D}_{1} \wedge\right.$ $\left.\cdots \wedge \mathcal{D}_{n}\right)=\operatorname{Sols}(\tau(\bar{x}))$.

- In step 5 , we have that if $\bar{t}_{0} \sqsubseteq \bar{t}_{i}$, for some cobasic set comp $\left(\bar{t}_{i}\right)$, then $\operatorname{Den}\left(\bar{t}_{0}\right) \subseteq \operatorname{Den}\left(\bar{t}_{i}\right)$ and $\operatorname{Den}\left(\bar{t}_{0} \otimes \operatorname{comp}\left(\bar{t}_{i}\right)\right)=\operatorname{Den}\left(\bar{t}_{0}\right) \cap \operatorname{Den}\left(\operatorname{comp}\left(\bar{t}_{i}\right)\right)=$ $\emptyset=\operatorname{Sols}\left(\mathcal{E} \wedge \mathcal{D}_{i}\right)$. Thus, $\operatorname{Den}(\beta)=\emptyset=\operatorname{Sols}(\tau(\bar{x}))$.

- In step 6 , if $\bar{t}_{i} \sqsubseteq \bar{t}_{j}$, then $\operatorname{Den}\left(\operatorname{comp}\left(\bar{t}_{i}\right)\right) \subseteq \operatorname{Den}\left(\operatorname{comp}\left(\bar{t}_{j}\right)\right)$ and $\operatorname{Den}\left(\bar{t}_{0} \otimes\right.$ $\left.\operatorname{comp}\left(\bar{t}_{i}\right)\right) \cap \operatorname{Den}\left(\bar{t}_{0} \otimes \operatorname{comp}\left(\bar{t}_{j}\right)\right)=\operatorname{Den}\left(\bar{t}_{0} \otimes \operatorname{comp}\left(\bar{t}_{j}\right)\right)$, thus $\operatorname{Den}(\beta)=$ $\operatorname{Den}\left(\beta^{\prime}\right)$.

\section{Example 4.3}

In order to illustrate the construction of minsets, we have created the program below, instead of using the previous quicksort program or a real application. This program exhibits features that can appear in different real cases, and thus allows us to illustrate almost all cases of the algorithm using a single example.

$$
\begin{aligned}
& \mathrm{p}(\mathrm{X} 1, \mathrm{X} 2, \mathrm{X} 3):-\mathrm{X} 1=\mathrm{f}(\mathrm{Y} 1, \mathrm{Y} 2), \mathrm{Y} 1 \neq \mathrm{r}(\mathrm{Z1}), \mathrm{Y} 2 \neq \mathrm{s}(\mathrm{Z} 2) \| \mathrm{X} 3=\mathrm{a} . \\
& \mathrm{p}(\mathrm{X} 1, \mathrm{X} 2, \mathrm{X} 3):-\mathrm{X} 1=\mathrm{f}(\mathrm{Y} 1, \mathrm{Y} 2), \mathrm{Y} 1=\mathrm{s}(\mathrm{Z} 1), \mathrm{Y} 2 \neq \mathrm{r}(\mathrm{Z} 2) \| \mathrm{X} 3=\mathrm{b} .
\end{aligned}
$$

The guarded program assumes a mode in which the first two arguments of $\mathrm{p} / 3$ are ground and the third one is free. Let the calling type be $\left(\alpha_{1}, \alpha_{1},-\right)$, where the type symbols $\alpha_{1}$ and $\alpha_{2}$ are defined as follows:

$$
\alpha_{1} \rightarrow\left\{f\left(\alpha_{2}, \alpha_{2}\right), g\left(\alpha_{2}, \alpha_{2}\right)\right\} \quad \alpha_{2} \rightarrow\{r(\mu), s(\mu)\}
$$

Let us take $\tau(\bar{x})$ in testgminset $(\tau(\bar{x}))$ to be the test of the first clause of $\mathrm{p} / 3$. That is, $\bar{x}=(\mathrm{X} 1, \mathrm{X} 2)$ and $\tau(\bar{x})=\tau(\mathrm{X} 1, \mathrm{X} 2) \equiv \mathrm{X} 1=\mathrm{f}(\mathrm{Y} 1, \mathrm{Y} 2) \wedge \mathrm{Y} 1$ $\neq \mathrm{r}(\mathrm{Z} 1) \wedge \mathrm{Y} 2 \neq \mathbf{s}(\mathrm{Z} 2)$. We have that $\tau(\mathrm{X} 1, \mathrm{X} 2)$ is written as $\mathcal{E} \wedge \mathcal{D}_{1} \wedge \mathcal{D}_{2}$, where $\mathcal{E} \equiv \mathrm{X} 1=\mathrm{f}(\mathrm{Y} 1, \mathrm{Y} 2), \mathcal{D}_{1} \equiv \mathrm{Y} 1 \neq \mathrm{r}(\mathrm{Z} 1)$ and $\mathcal{D}_{2} \equiv \mathrm{Y} 2 \neq \mathrm{s}(\mathrm{Z} 2)$. The minset $\beta$ which represents $\tau(\mathrm{X} 1, \mathrm{X} 2)$ is computed as follows:

1. $\theta_{0}=\{\mathrm{X} 1=\mathrm{f}(\mathrm{Y} 1, \mathrm{Y} 2)\}$ 
2. $\theta_{1}=\{\mathrm{X} 1=\mathrm{f}(\mathrm{r}(\mathrm{Z} 1), \mathrm{Y} 2), \mathrm{Y} 1=\mathrm{r}(\mathrm{Z} 1)\}$ is the substitution associated with the solved form of $X 1=f(Y 1, Y 2) \wedge Y 1=r(Z 1)$, i.e., the system of equations $\mathcal{E} \wedge \mathcal{N}_{1}$, where $\mathcal{N}_{1}$ is the negation of $Y 1 \neq r(Z 1)$.

3. $\theta_{2}=\{\mathrm{X} 1=\mathrm{f}(\mathrm{Y} 1, \mathrm{~s}(\mathrm{Z} 2)), \mathrm{Y} 2=\mathrm{s}(\mathrm{Z} 2)\}$ is the substitution associated with the solved form of $X 1=f(Y 1, Y 2) \wedge Y 2=s(Z 2)$.

4. Applying $\theta_{0}$ to $(\mathrm{X} 1, \mathrm{X} 2)$, we obtain $\bar{t}_{0}$, i.e., ( $\left.\mathrm{f}(\mathrm{Y} 1, \mathrm{Y} 2), \mathrm{X} 2\right)$. Also, $\bar{x} \theta_{1}=\bar{t}_{1}=(\mathrm{f}(\mathrm{r}(\mathrm{Z} 1), \mathrm{Y} 2), \mathrm{X} 2)$ and $\bar{x} \theta_{2}=\bar{t}_{2}=(\mathrm{f}(\mathrm{Y} 1, \mathrm{~s}(\mathrm{Z} 2))$, $\mathrm{X} 2)$. Thus, $\left.\beta^{\prime}=(\mathrm{f}(\mathrm{Y} 1, \mathrm{Y} 2), \mathrm{X} 2) \otimes \operatorname{comp}(\mathrm{f}(\mathrm{r}(\mathrm{Z} 1), \mathrm{Y} 2), \mathrm{X} 2)\right) \otimes$ $\operatorname{comp}((\mathrm{f}(\mathrm{Y} 1, \mathrm{~s}(\mathrm{Z} 2)), \mathrm{X} 2))$.

5. Finally, the simplification steps does not remove any cobasic set from $\beta^{\prime}$, thus $\beta=\beta^{\prime}$.

If we apply the algorithm to the second clause, we obtain the minset: $(\mathrm{f}(\mathrm{s}(\mathrm{Z} 1), \mathrm{Y} 2), \mathrm{X} 2) \otimes \operatorname{comp}((\mathrm{f}(\mathrm{s}(\mathrm{Z} 1), \mathrm{r}(\mathrm{Z} 2)), \mathrm{X} 2))$.

\section{Definition 4.7 (type-annotated term instance)}

Let $\delta_{1}=\left(\bar{t}_{1}, \rho_{1}\right)$ and $\delta_{2}=\left(\bar{t}_{2}, \rho_{2}\right)$ be two type-annotated terms. We say that $\delta_{1}$ is an instance of $\delta_{2}$ if $\delta_{1} \sqsubseteq \delta_{2}$ and there is a substitution $\theta$ such that $\bar{t}_{1}=\bar{t}_{2} \theta$.

\section{Reduction of the Checking Exclusion Problem}

Let $\tau_{1}(\bar{x})$ and $\tau_{2}(\bar{x})$ be tests which are conjunctions of unification and disunification tests, and $\rho$ a type assignment. Let $\delta$ be a type-annotated term representing the type assignment $\rho$. Let $\beta_{i}$ be a minset representing $\tau_{i}$, for $i=1,2$, i.e., $\beta_{i}=$ test 2 minset $\left(\tau_{i}\right)$ (where the test2minset function is given in Definition 4.6). We have that $\tau_{1}(\bar{x})$ and $\tau_{2}(\bar{x})$ are exclusive w.r.t. $\rho$ if and only if $\delta \otimes \beta_{1} \otimes \beta_{2} \simeq \Lambda$. Let $\beta$ be the minset resulting of computing $\beta_{1} \otimes \beta_{2}$ (this intersection can be trivially defined in terms of most general unifiers of the tuples of terms composing the minsets $\beta_{1}$ and $\beta_{2}$ ). Then, the fundamental problem is to devise an algorithm to test whether $\delta \otimes \beta \simeq \Lambda$, where $\delta$ is a type-annotated term and $\beta$ a minset.

\section{Example 4.4}

Consider the mutual exclusion problem for the input tests and calling type given in Example 4.3 for predicate $\mathrm{p} / 3$. Such calling type is written as the type assignment $\left(\left(X 1, X_{2}\right):\left(\alpha_{1}, \alpha_{1}\right)\right)$, which is represented as the type-annotated term $\delta$, where $\delta=\left((X 1, X 2),\left(X 1: \alpha_{1}, X_{2}: \alpha_{1}\right)\right)$. The tests and minsets representing them respectively are:

$$
\begin{aligned}
& \tau_{1}(\bar{x})=\tau_{1}(\mathrm{X} 1, \mathrm{X} 2) \equiv \mathrm{X} 1=\mathrm{f}(\mathrm{Y} 1, \mathrm{Y} 2) \wedge \mathrm{Y} 1 \neq \mathrm{r}(\mathrm{Z} 1) \wedge \mathrm{Y} 2 \neq \mathrm{s}(\mathrm{Z} 2), \\
& \tau_{2}(\bar{x})=\tau_{2}(\mathrm{X} 1, \mathrm{X} 2) \equiv \mathrm{X} 1=\mathrm{f}(\mathrm{Y} 1, \mathrm{Y} 2) \wedge \mathrm{Y} 1=\mathrm{s}(\mathrm{Z} 1) \wedge \mathrm{Y} 2 \neq \mathrm{r}(\mathrm{Z} 2), \\
& \beta_{1}=(\mathrm{f}(\mathrm{Y} 1, \mathrm{Y} 2), \mathrm{X} 2) \otimes \operatorname{comp}((\mathrm{f}(\mathrm{r}(\mathrm{Z} 1), \mathrm{Y} 2), \mathrm{X} 2)) \otimes \operatorname{comp}((\mathrm{f}(\mathrm{Y} 1, \\
& \mathrm{s}(\mathrm{Z} 2)), \mathrm{X} 2)), \text { and } \\
& \beta_{2}=(\mathrm{f}(\mathrm{s}(\mathrm{Z} 1), \mathrm{Y} 2), \mathrm{X} 2) \otimes \operatorname{comp}((\mathrm{f}(\mathrm{s}(\mathrm{Z} 1), \mathrm{r}(\mathrm{Z} 2)), \mathrm{X} 2)) . \\
& \mathrm{Thus}, \beta \simeq \beta_{1} \otimes \beta_{2} \equiv\left(f\left(s\left(X_{3}\right), X_{4}\right), X_{5}\right) \otimes \operatorname{comp}\left(f\left(s\left(X_{6}\right), s\left(X_{7}\right)\right), X_{8}\right) \otimes \\
& \operatorname{comp}\left(f\left(s\left(X_{9}\right), r\left(X_{10}\right)\right), X_{11}\right) .
\end{aligned}
$$




\section{A High Level Description of the Algorithm}

We first provide a high level description of the algorithm that we propose, whose detailed description is given by the boolean function $\operatorname{empty}(\delta, \beta)$ in Definitions $4.13,4.14$ and $4.15:^{* 2}$

- First, perform the "intersection" of the type-annotated term $\delta$ and the tuple of terms $\bar{t}_{0}$ of the minset $\beta$ (we assume that $\beta=\bar{t}_{0} \otimes \mathcal{C}$ and that $\beta \not \Lambda$ ). Let $\delta^{\prime}$ denote the type-annotated term resulting from this intersection (i.e., $\left.\delta^{\prime}=\delta \otimes \bar{t}_{0}\right)$. This operation is implemented by the intersec $\left(\delta, \bar{t}_{0}\right)$ function described in Definition 4.10 (recall that a tuple of terms is a typeannotated term). Consider for example $\delta$ and $\beta$ given in Example 4.4. In this case, $\bar{t}_{0}$ denotes the tuple of terms $\left(f\left(s\left(X_{3}\right), X_{4}\right), X_{5}\right)$ and $\mathcal{C}$ denotes the set of cobasic sets $\left\{\operatorname{comp}\left(f\left(s\left(X_{6}\right), s\left(X_{7}\right)\right), X_{8}\right), \operatorname{comp}\left(f\left(s\left(X_{9}\right), r\left(X_{10}\right)\right)\right.\right.$, $\left.\left.X_{11}\right)\right\}$. Thus, the "intersection" of $\delta$ and $\bar{t}_{0}$ is the type-annotated term $\delta^{\prime}=\left(\left(f\left(s\left(X_{12}\right), X_{13}\right), X_{14}\right),\left(X_{12}: \mu, X_{13}: \alpha_{2}, X_{14}: \alpha_{1}\right)\right.$.

- If $\delta^{\prime}$ is empty (i.e., $\delta^{\prime} \simeq \Lambda$ ), then it can be reported that $\delta \otimes \beta \simeq \Lambda$. Otherwise, if $\bar{t}_{0}$ is "included" in $\delta^{\prime}$ (i.e., $\bar{t}_{0} \sqsubseteq \delta^{\prime}$ ), then it can be reported that $\delta \otimes \beta \not \Lambda$ (note that it always holds that $\beta \sqsubseteq \bar{t}_{0}$ ). The "inclusion" operation can be defined by using a straightforward adaptation of the $\operatorname{subset}_{T}\left(\omega_{1}, \omega_{2}\right)$ function described in ${ }^{4)}$, that determines whether the type denoted by a pure type term (a variable-free type term) is a subset of the type denoted by another. We denote our "inclusion" operation by the included $\left(\delta_{1}, \delta_{2}\right)$ function, which returns true if and only if $\delta_{1} \sqsubseteq \delta_{2}$, where $\delta_{1}$ and $\delta_{2}$ are type-annotated terms. In our example, none of these conditions hold (recall that the tuple of terms $\left(f\left(s\left(X_{3}\right), X_{4}\right), X_{5}\right)$ represents the type-annotated term $\left(\left(f\left(s\left(X_{3}\right), X_{4}\right), X_{5}\right),\left(X_{3}: \mu, X_{4}: \mu, X_{5}: \mu\right)\right)$, and that a type-annotated term can be trivially rewritten as a pure type term).

- Otherwise, the problem is reduced to checking whether $\delta^{\prime} \otimes \mathcal{C} \simeq \Lambda$ (this is done by the auxiliary function empty1, described in detail in Definition 4.14). Note that $\delta^{\prime} \otimes \mathcal{C}$ can be seen as a system of one equation (corresponding to $\delta^{\prime}$ ) and zero or more disequations (each of them corresponding to a cobasic set in $\mathcal{C}$ ). Thus, the problem can be seen as determining whether such system has no solutions.

- This way, if $\delta^{\prime}$ is "included" in the tuple of terms of some cobasic set in $\mathcal{C}$, then it can be reported that $\delta^{\prime} \otimes \mathcal{C} \simeq \Lambda$.

- Otherwise, it means that $\delta^{\prime}$ is "too big", and thus, it is "expanded" to a set of "smaller" type-annotated terms (with the hope that each of them will be "included" in the tuple of terms of some cobasic set in $\mathcal{C}$ ). This way, the initial problem is reduced to a finite number of subproblems, one subproblem for each element in the set of "smaller" type-annotated terms to which $\delta^{\prime}$ has been "expanded". This holds in the example,

*2 We use the type representation of ${ }^{4)}$, and assume that there is a common set of rules where type symbols are described. For brevity, we omit such set of type rules in the description of the algorithms. 
where the type-annotated term $\delta^{\prime}$ is "expanded" to a set of two "smaller" type-annotated terms $\left\{\delta_{1}^{\prime}, \delta_{2}^{\prime}\right\}$ (expanding variable $X_{13}$ ) where $\delta_{1}^{\prime}$ denotes $\left(\left(f\left(s\left(X_{15}\right), r\left(X_{16}\right)\right), X_{17}\right),\left(X_{15}: \mu, X_{16}: \mu, X_{17}: \alpha_{1}\right)\right)$ and $\delta_{2}^{\prime}$ denotes $\left(\left(f\left(s\left(X_{18}\right), s\left(X_{19}\right)\right), X_{20}\right),\left(X_{18}: \mu, X_{19}: \mu, X_{20}: \alpha_{1}\right)\right)$. Then, two subproblems arise:

- Checking whether $\delta_{1}^{\prime} \otimes \operatorname{comp}\left(f\left(s\left(X_{6}\right), r\left(X_{7}\right)\right), X_{8}\right) \simeq \Lambda$, which holds because $\delta_{1}^{\prime}$ is "included" in $\left(f\left(s\left(X_{6}\right), r\left(X_{7}\right)\right), X_{8}\right)$, the tuple of terms of the cobasic set $\operatorname{comp}\left(f\left(s\left(X_{6}\right), r\left(X_{7}\right)\right), X_{8}\right)$; and

- Checking whether $\delta_{2}^{\prime} \otimes \operatorname{comp}\left(f\left(s\left(X_{9}\right), r\left(X_{10}\right)\right), X_{11}\right) \simeq \Lambda$ is empty, which also holds because $\delta_{2}^{\prime}$ is "included" in $\left(f\left(s\left(X_{9}\right), r\left(X_{10}\right)\right), X_{11}\right)$.

- Thus, it can be concluded that $\delta^{\prime} \otimes \mathcal{C} \simeq \Lambda$ and hence $\delta \otimes \beta \simeq \Lambda$.

Termination of this algorithm is granted because a) the original problem is divided into a finite number of subproblems, since the type-annotated term of the problem is expanded into a finite number of type-annotated terms, each one giving rise to a subproblem, b) the number of cobasic sets in the initial problem is finite, and c) the number of cobasic sets decreases at least by one in each subproblem (recursive call).

\section{A Detailed Description of the Algorithm}

The detailed description of the empty $(\delta, \beta)$ function requires some (instrumental) definitions, namely the definition of "useless" cobasic set and the aliased, intersec, and expansion functions.

\section{Definition 4.8 (useless cobasic set)}

Given a type-annotated term $\delta$, a set of cobasic sets $\mathcal{C}$, and a cobasic set $\operatorname{comp}(\bar{t}) \in \mathcal{C}$, we say that $\operatorname{comp}(\bar{t})$ is useless for determining whether $\delta \otimes \mathcal{C} \simeq \Lambda$, whenever if $\delta \otimes(\mathcal{C}-\{\operatorname{comp}(\bar{t})\}) \not 4 \Lambda$, then $\delta \otimes \mathcal{C} \neq \Lambda$ (or, equivalently, if $\delta \otimes \mathcal{C} \simeq \Lambda$, then $\delta \otimes(\mathcal{C}-\{\operatorname{comp}(\bar{t})\}) \simeq \Lambda)$.

It is easy to prove that the reciprocal also holds. If $\delta \otimes(\mathcal{C}-\{\operatorname{comp}(\bar{t})\}) \simeq$ $\Lambda$, then obviously $\delta \otimes \mathcal{C} \simeq \Lambda$ (note that $(\delta \otimes \mathcal{C}) \sqsubseteq(\delta \otimes(\mathcal{C}-\{\operatorname{comp}(\bar{t})\}))$ ). Thus, if $\operatorname{comp}(\bar{t}) \in \mathcal{C}$ is an useless cobasic set, then $\delta \otimes \mathcal{C} \simeq \Lambda$ if and only if $\delta \otimes(\mathcal{C}-\{\operatorname{comp}(\bar{t})\}) \simeq \Lambda$.

Definition 4.9 (aliased $(\delta, \bar{t})$ function)

Let $\delta$ be the type-annotated term $\left(\bar{t}_{\delta}, \rho_{\delta}\right), \delta \neq \Lambda, \bar{t}$ a tuple of terms, and $\theta=$ $m g u\left(\bar{t}_{\delta}, \bar{t}\right)$. We define the aliased function as follows:

$$
\begin{aligned}
\operatorname{aliased}(\delta, \bar{t})= & \left\{x \in \operatorname{vars}\left(\bar{t}_{\delta}\right) \mid x \theta \text { is a variable, and exists } x^{\prime} \in \operatorname{vars}\left(\bar{t}_{\delta}\right),\right. \\
& \left.x \neq x^{\prime}, \text { such that } x \theta=x^{\prime} \theta\right\} .
\end{aligned}
$$

Given a type-annotated term $\delta$ and a tuple of terms $\bar{t}$, the intersec $(\delta, \bar{t})$ function returns a type-annotated term whose meaning is the same as $\delta \otimes \bar{t}$ (recall that a tuple of terms is also a type-annotated term). This function can be defined as a straightforward adaptation of the unify $\left(\omega_{1}, \omega_{2}, T, \Theta\right)$ function 
described in ${ }^{4)}$, that performs a type unification, where $\omega_{1}$ and $\omega_{2}$ are the type terms to be unified, $\Theta$ a type substitution for the variables in $\omega_{1}$ and $\omega_{2}$, and $T$ a set of type rules defining the type symbols appearing in $\omega_{1} \Theta, \omega_{2} \Theta$, and $\Theta$. The output of the function unify is a triple $\left(\omega_{f}, T_{f}, \Theta_{f}\right)$, where $\omega_{f}$ is a type term, $\Theta_{f}$ a type substitution for the variables in $\omega_{f}$, and $T_{f}$ a set of type rules defining the type symbols appearing in the pure type term $\omega_{f} \Theta_{f}$, such that $T \subseteq T_{f}$. Since type terms can be trivially rewritten as type-annotated terms, we can define function intersec $(\delta, \bar{t})$ as follows:

Definition 4.10 (intersec $(\delta, \bar{t})$ function)

Given a type-annotated term $\delta$ and a tuple of terms $\bar{t}$, the process of function intersec $(\delta, \bar{t})$ is:

- Let $\delta$ be the pair $\left(\bar{t}_{\delta}, \rho_{\delta}\right)$, and $\left(\omega_{f}, T_{f}, \Theta_{f}\right)=$ unify $\left(\bar{t}_{\delta}, \bar{t}, T, \Theta\right)$ (note that a tuple of terms is a particular case of type term, and that $\bar{t}_{\delta}$ and $\bar{t}$ are tuples of terms), where $\Theta$ is a type substitution constructed as follows:

$$
x \Theta= \begin{cases}\omega & \text { if } x \in \operatorname{vars}(\delta) \text { and } \rho_{\delta}(x)=\omega \\ x & \text { otherwise. }\end{cases}
$$

and $T$ a set of type rules defining the type symbols in $\bar{t}_{\delta} \Theta$.

- Rewrite $\omega_{f} \Theta_{f}$ as a type-annotated term $\delta^{\prime}$ and return it. For simplicity, we assume that the function returns only a type-annotated term $\delta^{\prime}$, but in fact, it returns a pair $\left(\delta^{\prime}, T_{f}\right)$, where $T_{f}$ is a set of type rules defining the type symbols appearing in $\delta^{\prime}$.

\section{Theorem 4.2}

Given a type-annotated term $\delta$ and a tuple of terms $\bar{t}$, then: (i) intersec $(\delta, \bar{t})$ terminates, (ii) intersec $(\delta, \bar{t}) \simeq \delta \otimes \bar{t}$, and (iii) $\operatorname{intersec}(\delta, \bar{t})=\Lambda$ iff $\delta \otimes \bar{t} \simeq \Lambda$.

\section{Proof}

It follows from Theorem 5.60 of ${ }^{4)}$, since the function intersec is an adaptation of the function unify $\left(\omega_{1}, \omega_{2}, T, \Theta\right)$.

The expansion of a type-annotated term into a set of smaller type-annotated terms is performed by the expansion function defined below.

Definition 4.11 (expansion $(\delta, \operatorname{comp}(\bar{t}))$ function)

Let $\delta$ be a type-annotated term $\left(\bar{t}_{\delta}, \rho_{\delta}\right), \delta \not \Lambda$, and comp $(\bar{t})$ a cobasic set such that $\delta \otimes \bar{t} \not \Lambda$ and $\delta \nsubseteq \bar{t}$. We also assume that $\operatorname{vars}\left(t_{\delta}\right) \cap \operatorname{vars}(\bar{t})=\emptyset$. The function expansion $(\delta, \operatorname{comp}(\bar{t}))$ returns a pair $\left(\delta^{\prime}, \Delta\right)$ which is a "partition" of $\delta$, i.e.:

- $\delta^{\prime}$ is a type-annotated term instance of $\delta,\left(\bar{t}_{\delta^{\prime}}, \rho_{\delta^{\prime}}\right), \delta^{\prime} \not \subset \Lambda . \delta^{\prime}$ is obtained by expanding $\delta$ to some "decision depth" that allows to detect if the cobasic set comp $(\bar{t})$ is useless (see Definition 4.8 of useless cobasic set);

- $\Delta$ is a set of type-annotated terms;

- for all $x \in \operatorname{vars}\left(\delta^{\prime}\right)$, it holds that: $\rho_{\delta^{\prime}}(x)=\mu, \rho_{\delta}(x)$ is a base type symbol, or $x \theta$ is a variable, where $\theta$ is the most general unifier of $\bar{t}_{\delta^{\prime}}$ and $\bar{t}$ (note 
that the variables of $\delta$ whose type is $\mu$ or a base type are not "expanded");

- $\left(\cup_{\delta^{\prime \prime} \in \Delta} \operatorname{Den}\left(\delta^{\prime \prime}\right)\right) \cup \operatorname{Den}\left(\delta^{\prime}\right)=\operatorname{Den}(\delta)$ (i.e., $\left.\delta \simeq\left(\bigoplus_{\delta^{\prime \prime} \in \Delta} \delta^{\prime \prime}\right) \oplus \delta^{\prime}\right)$; and

- for all $\delta^{\prime \prime} \in \Delta, \delta^{\prime \prime} \otimes \bar{t} \simeq \Lambda$ (this is ensured because type rules are deterministic).

We define the expansion function as:

$$
\operatorname{expansion}(\delta, \operatorname{comp}(\bar{t}))=\operatorname{expands}\left(\operatorname{var} s\left(t_{\delta}\right), m g u\left(\bar{t}_{\delta}, \bar{t}\right), \delta, \emptyset\right)
$$

where the expands function is defined below:

Definition 4.12 (expands $(V, \theta, \delta, \Delta)$ function)

Let $\delta$ be a type-annotated term $\left(\bar{t}_{\delta}, \rho_{\delta}\right), V$ a set of variables $V \subseteq \operatorname{vars}\left(t_{\delta}\right), \theta$ a substitution for the variables in $t_{\delta}$, and $\Delta$ a set of type-annotated terms which are pairwise disjoint and disjoint with $\delta$. The expands function returns a pair $\left(\delta^{\prime}, \Delta^{\prime}\right)$, where $\delta^{\prime}$ is a type-annotated term and $\Delta^{\prime}$ a set of type-annotated terms, and is defined by the following algorithm:

1: $V^{\prime} \leftarrow\left\{x \in V \mid x \theta\right.$ is not a variable, $\rho_{\delta}(x) \neq \mu$, and $\rho_{\delta}(x)$ is not a base type symbol $\}$

2: if $V^{\prime}=\emptyset$ then return the pair $(\delta, \Delta)$

3: else

4: $\quad$ Take a variable $x \in V^{\prime}$

5: Let $x \theta=f\left(t_{1}, \ldots, t_{n}\right)$, where $n \geq 0$ and $t_{1}, \ldots, t_{n}$ are terms (if $x \theta$ is a constant, it is treated as the particular case where $n=0$ ).

6: $\quad \alpha \leftarrow \rho_{\delta}(x)$

7: $\quad$ if $\alpha$ is a type symbol then

8: $\quad$ Let the type rule defining $\alpha$ be $\alpha \rightarrow \Upsilon$

9: $\quad$ Let $\omega \in \Upsilon$, such that $\omega=f\left(\alpha_{1}, \ldots, \alpha_{n}\right)$, i.e., $\omega$ has the same principal function symbol (and arity) than $x \theta$ (note that such $\omega$ always exists, since $\delta \otimes \bar{t} \not \Lambda$, and type rules are deterministic.)

10: $\quad \Upsilon^{\prime} \leftarrow \Upsilon-\{\omega\}$

11: else (necessarily $\alpha$ is a pure type term of the form $f\left(\alpha_{1}, \ldots, \alpha_{n}\right)$ )

12: $\quad \omega \leftarrow \alpha$

13: $\quad \Upsilon^{\prime} \leftarrow \emptyset$

14: end if

15: $\bar{t}_{\delta}^{\prime} \leftarrow \bar{t}_{\delta}\left[x / f\left(y_{1}, \ldots, y_{n}\right)\right]$, where $y_{1}, \ldots, y_{n}$ are new and distinct variables, and $\bar{t}_{\delta}[y / t]$ denotes the instance of the tuple of terms $\bar{t}_{\delta}$ obtained by substituting all occurrences of variable $y$ by term $t$ in $\bar{t}_{\delta}$

16: $\rho_{\delta}^{\prime} \leftarrow\left(\rho_{\delta}-\{x: \alpha\}\right) \cup\left\{y_{1}: \alpha_{1}, \ldots, y_{n}: \alpha_{n}\right\}$, i.e., $\rho_{\delta}^{\prime}$ is the type assignment obtained by removing $x: \alpha$ from $\rho_{\delta}$, and adding $y_{1}: \alpha_{1}, \ldots, y_{n}: \alpha_{n}$ to the result

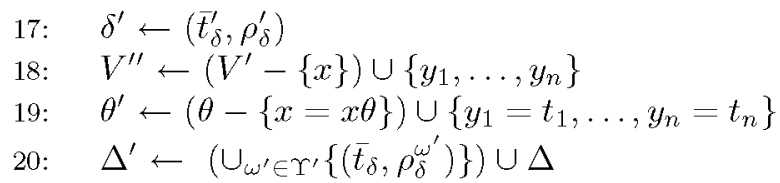


where $\rho_{\delta}^{\omega^{\prime}}$ is the type assignment obtained by removing $x: \alpha$ from $\rho_{\delta}$, and adding $x: \omega^{\prime}$ to $\rho_{\delta}$.

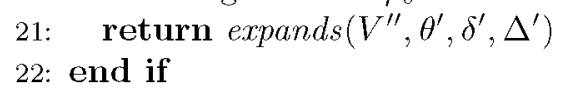

\section{Example 4.5}

Reconsider the type annotated term:

$$
\delta^{\prime}=\left(\left(f\left(s\left(X_{12}\right), X_{13}\right), X_{14}\right),\left(X_{12}: \mu, X_{13}: \alpha_{2}, X_{14}: \alpha_{1}\right)\right.
$$

and the set of cobasic sets:

$$
\mathcal{C}=\left\{\operatorname{comp}\left(f\left(s\left(X_{6}\right), s\left(X_{7}\right)\right), X_{8}\right), \operatorname{comp}\left(f\left(s\left(X_{9}\right), r\left(X_{10}\right)\right), X_{11}\right)\right\}
$$

of Example 4.4 .

Let us choose the cobasic set $\operatorname{comp}(\bar{t})=\operatorname{comp}\left(f\left(s\left(X_{6}\right), s\left(X_{7}\right)\right), X_{8}\right)$ from $\mathcal{C}$ (note that $\delta^{\prime}$ is not included in the tuple of terms $\left.\bar{t}=\left(f\left(s\left(X_{6}\right), s\left(X_{7}\right)\right), X_{8}\right)\right)$. We expand $\delta^{\prime}$ by calling the expansion function (in Definition 4.11) as follows:

$\operatorname{expansion}\left(\delta^{\prime}, \operatorname{comp}(\bar{t})\right)=\operatorname{expands}\left(\operatorname{var} s\left(t_{\delta^{\prime}}\right), \operatorname{mgu}\left(\bar{t}_{\delta^{\prime}}, \bar{t}\right), \delta^{\prime}, \emptyset\right)=$ expands $\left(\left\{X_{12}, X_{13}, X_{14}\right\},\left\{X_{12}=X_{6}, X_{13}=s\left(X_{7}\right), X_{14}=X_{8}\right\}, \delta^{\prime}, \emptyset\right)=\left(\delta_{1}, \Delta_{1}\right)$

where $\delta_{1}=\left(\left(f\left(s\left(X_{15}\right), r\left(X_{16}\right)\right), X_{17}\right),\left(X_{15}: \mu, X_{16}: \mu, X_{17}: \alpha_{1}\right)\right)$ and $\Delta_{1}=\left\{\left(\left(f\left(s\left(X_{18}\right), s\left(X_{19}\right)\right), X_{20}\right),\left(X_{18}: \mu, X_{19}: \mu, X_{20}: \alpha_{1}\right)\right)\right\}$. This is done by choosing variable $X_{13}$ in step 4.1 of the expands function (see Definition 4.12 ) and using its type, $\alpha_{2}$, in step 4.1 .

Definition 4.13 (empty $(\delta, \beta)$ function)

Given a type-annotated term $\delta$ and a minset $\beta$ such that $\beta \neq \Lambda\left(\beta=\bar{t}_{0} \otimes \mathcal{C}\right.$, where $\bar{t}_{0}$ is a tuple of terms, and $\mathcal{C}$ a set of cobasic sets), we define:

$$
\operatorname{empty}(\delta, \beta)= \begin{cases}\text { true } & \text { if } \delta^{\prime}=\Lambda \\ \text { false } & \text { if included }\left(\bar{t}_{0}, \delta^{\prime}\right) \\ \text { empty1 }\left(\mathcal{C}, \delta^{\prime}, \emptyset\right) & \text { otherwise }\end{cases}
$$

where $\delta^{\prime}=$ intersec $\left(\delta, \bar{t}_{0}\right)$.

Definition 4.14 (empty $1(\mathcal{C}, \delta, \Gamma)$ function)

Given a type-annotated term $\delta$ (i.e., a pair $\left(\bar{t}_{\delta}, \rho_{\delta}\right)$ ) such that $\delta \neq \Lambda$, a set of cobasic sets $\mathcal{C}$, and a set $\Gamma$ of triples of the form $\left(\delta_{1}, \mathcal{V}, \operatorname{comp}(\bar{t})\right)$ where:

- $\delta_{1}$ is a type-annotated term $\delta_{1}=\left(\bar{t}_{1}, \rho_{1}\right)$, such that $\delta_{1} \neq \Lambda$,

- $\operatorname{comp}(\bar{t})$ is a cobasic set,

- $\operatorname{vars}\left(\delta_{1}\right) \cap \operatorname{vars}(\operatorname{comp}(\bar{t}))=\emptyset$,

- $\theta=m g u\left(\bar{t}_{1}, \bar{t}\right)$,

- for all $x \in \operatorname{var} s\left(\delta_{1}\right), x \theta$ is a variable, and

- $v \in \mathcal{V}$ iff $v \in \operatorname{var} s\left(\delta_{1}\right), \rho_{\delta_{1}}(v) \neq \mu, \rho_{\delta_{1}}(v)$ is not a base type symbol, and $\exists v^{\prime} \in \operatorname{var} s\left(\delta_{1}\right), v \neq v^{\prime}$, such that $v \theta=v^{\prime} \theta$ (i.e., $\mathcal{V}$ is the set of variables in $\operatorname{var} s\left(\delta_{1}\right)$ which are aliased with some other variable in $\operatorname{vars}\left(\delta_{1}\right)$ by $\left.\theta\right)$. 
we define the empty1 function in Algorithm 1.

The empty $1(\mathcal{C}, \delta, \Gamma)$ function performs a "first pass" over the cobasic sets in $\mathcal{C}$. This pass results in the removal of cobasic sets that are inferred to be useless. Some useless cobasic sets are removed in step 1: if intersec $(\delta, \bar{t})=\Lambda$, for some $\operatorname{comp}(\bar{t}) \in \mathcal{C}$, then $\operatorname{comp}(\bar{t})$ is useless for determining whether $\delta \otimes \mathcal{C} \simeq \Lambda$, because none of the instances of $\delta$ meet the equality constraint imposed by $\bar{t}$, and hence all the instances of $\delta$ meet the inequality constraint imposed by $\operatorname{comp}(\bar{t})$. Thus, $\delta \otimes \mathcal{C} \simeq \Lambda$ if and only if the rest of cobasic sets, $\mathcal{C}-\{\operatorname{comp}(\bar{t})\}$, impose (inequality) constraints that are not met by any instance of $\delta$. If $\operatorname{included}(\delta, \bar{t})$ for some cobasic set $\operatorname{comp}(\bar{t})$ in $\mathcal{C}^{\prime}$ (as it is checked in step 6), then all the instances of $\delta$ meet the equality constraint imposed by $\bar{t}$, and hence, none of the instances of $\delta$ meet the inequality constraint imposed by $\operatorname{comp}(\bar{t})$. Thus, in this case, $\delta \otimes \mathcal{C} \simeq \Lambda$. The rationale behind steps 9 to 11 is that at this point (where not included $(\delta, \bar{t})$ nor intersec $(\delta, \bar{t})=\Lambda) \delta$ is "too big," and thus it is "expanded" to a set of

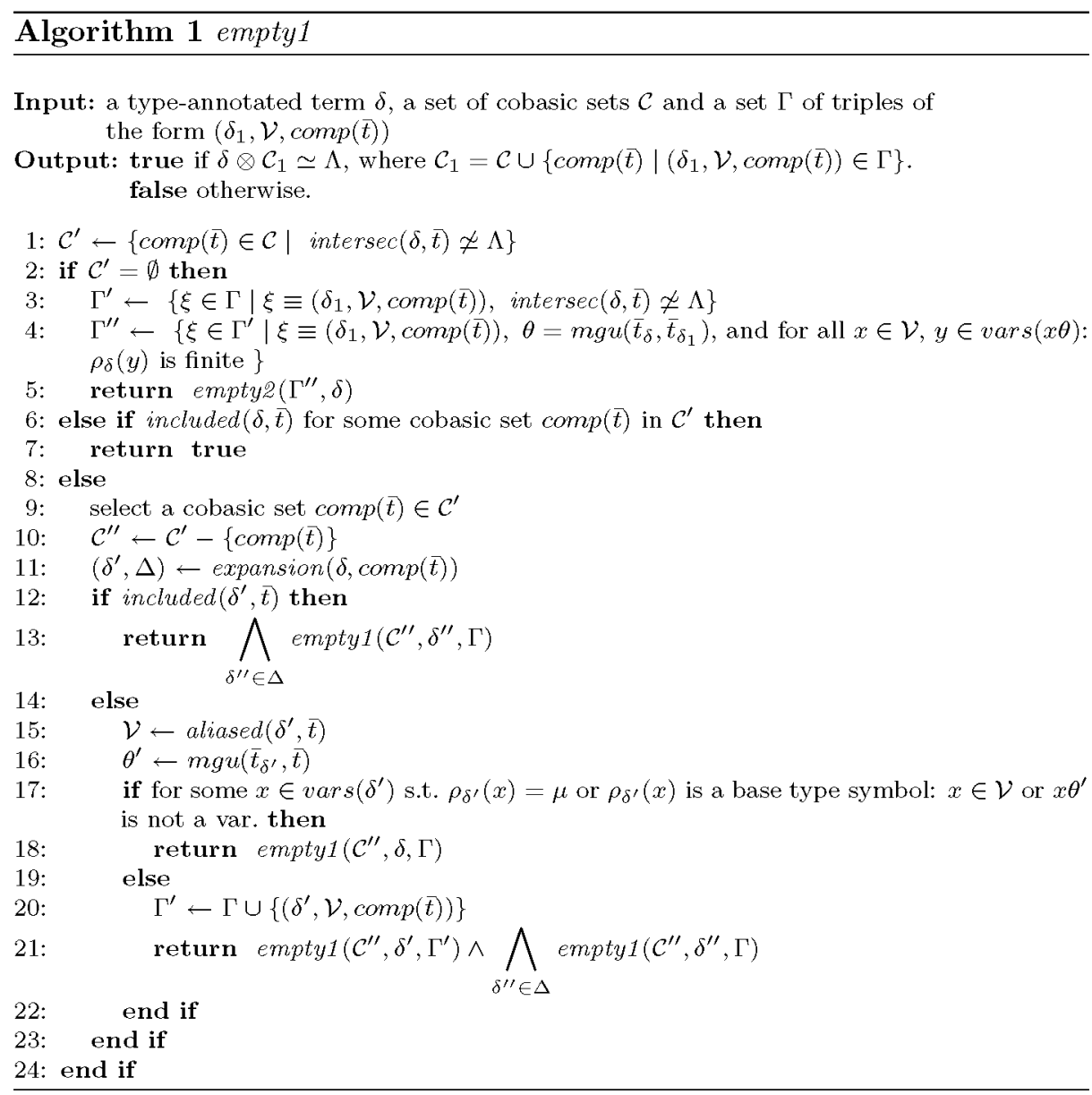


"smaller" type-annotated terms $\left\{\delta^{\prime}\right\} \cup \Delta$ (using the expansion function given in definition 4.11), in the hope that each of them will be "included" in the tuple of terms of some cobasic set in $\mathcal{C}^{\prime}$. In this expansion, $\delta^{\prime}$ is obtained by expanding variables $v \in \operatorname{vars}(\delta)$ to at most a depth given by $v \sigma$, where $\sigma=m g u\left(\bar{t}_{\delta}, \bar{t}\right)$. When inclusion is checked at step 12 , if included $\left(\delta^{\prime}, \bar{t}\right)$, then necessarily for all $x \in \operatorname{vars}\left(\delta^{\prime}\right)$ it holds that $x \theta^{\prime}$ is a variable, where $\theta^{\prime}=m g u\left(\bar{t}_{\delta^{\prime}}, \bar{t}\right)$ (step 16). In this case, $\operatorname{comp}(\bar{t})$ is not considered in the recursive calls in step 13 , since (according to definition 4.11) for all $\delta^{\prime \prime} \in \Delta, \delta^{\prime \prime} \otimes \bar{t} \simeq \Lambda$, and thus, $\operatorname{comp}(\bar{t})$ is useless for all of these subproblems. If not included $\left(\delta^{\prime}, \bar{t}\right)$, then: a) $\bar{t}$ imposes some equality constraints over some variables in $\delta$ (such variables are gathered together in step 15, where the set $\mathcal{V}$ is created using the aliased function given in Definition 4.9 ), or b) $\bar{t}$ restricts the values of some variable(s) in $\delta^{\prime}$ whose type is $\mu$, unifying them to some term (which is not a variable). If the condition checked at step 17 holds, then there is always an instance of $\delta^{\prime}$ which does not meet the former constraints a) or b), and thus $\operatorname{comp}(\bar{t})$ is useless. In step 20, cobasic sets which are not deemed useless at this point are stored in $\Gamma$, which is an accumulation parameter. $\delta^{\prime}$ and $\mathcal{V}$ (besides comp $(\bar{t})$ ) are recorded in this parameter, because aliased variables whose type is infinite (or which after having been expanded are bound to a term containing variables whose type is infinite) allow us to detect useless cobasic sets, since it is always possible to find an instance of $\delta^{\prime}$ which does not meet the equality constraints imposed by $\bar{t}$ (case a)). Useless cobasic sets are then subsequently removed in steps 3 and 4 , before empty $2\left(\Gamma^{\prime}, \delta\right)$ is called in step 5 . The first pass over the cobasic sets ends in step 2 when condition $\mathcal{C}^{\prime}=\emptyset$ holds. Note that when this condition holds, step 4 checks that a type expression denotes a finite set of terms, and there are straightforward algorithms to test this. The empty2 function performs a second pass over the remaining cobasic sets, checking whether the constraints described previously in case a) hold. Since the types of the variables involved in such constraints are finite (i.e., they represent finite sets of terms), the process performed by the empty2 function is simple, sound, complete, and terminating.

Definition 4.15 (empty2 $(\Gamma, \delta)$ function)

Given a type-annotated term $\delta$, such that $\delta \neq \Lambda$, and a set $\Gamma$ of triples of the form $\left(\delta_{1}, \mathcal{V}, \operatorname{comp}(\bar{t})\right)$ similar to those in the third parameter of the function empty $1(\mathcal{C}, \delta, \Gamma)$ in definition 4.14 , but with the following additional constraint:

for all $x \in \mathcal{V}, \rho_{\delta_{1}}(x)$ is finite (note that for all $v \in \operatorname{var} s\left(\delta_{1}\right)$ such that

$v \notin \mathcal{V}, \rho_{\delta_{1}}(v)$ can be any type, including $\mu$ or a base type symbol),

we define the function empty2 in Algorithm 2, where $\delta[y / t]$ denotes an instance of type annotated term $\delta$ obtained by substituting variable $y$ by term $t$.

The function empty2 $(\Gamma, \delta)$ selects a cobasic set $\operatorname{comp}(\bar{t})$ in $\Gamma$, and, if $\delta$ is not included in $\bar{t}$, then $\delta$ is expanded (in step 13) to a set of type-annotated terms $\Delta$ by substituting only "decision variables." Such expansion ensures that every $\delta^{\prime \prime} \in \Delta$ is either "included" in $\bar{t}$ or "disjoint" with it. It also ensures that $\delta$ is not infinitely expanded: the type of such variables is finite. Note that, at 


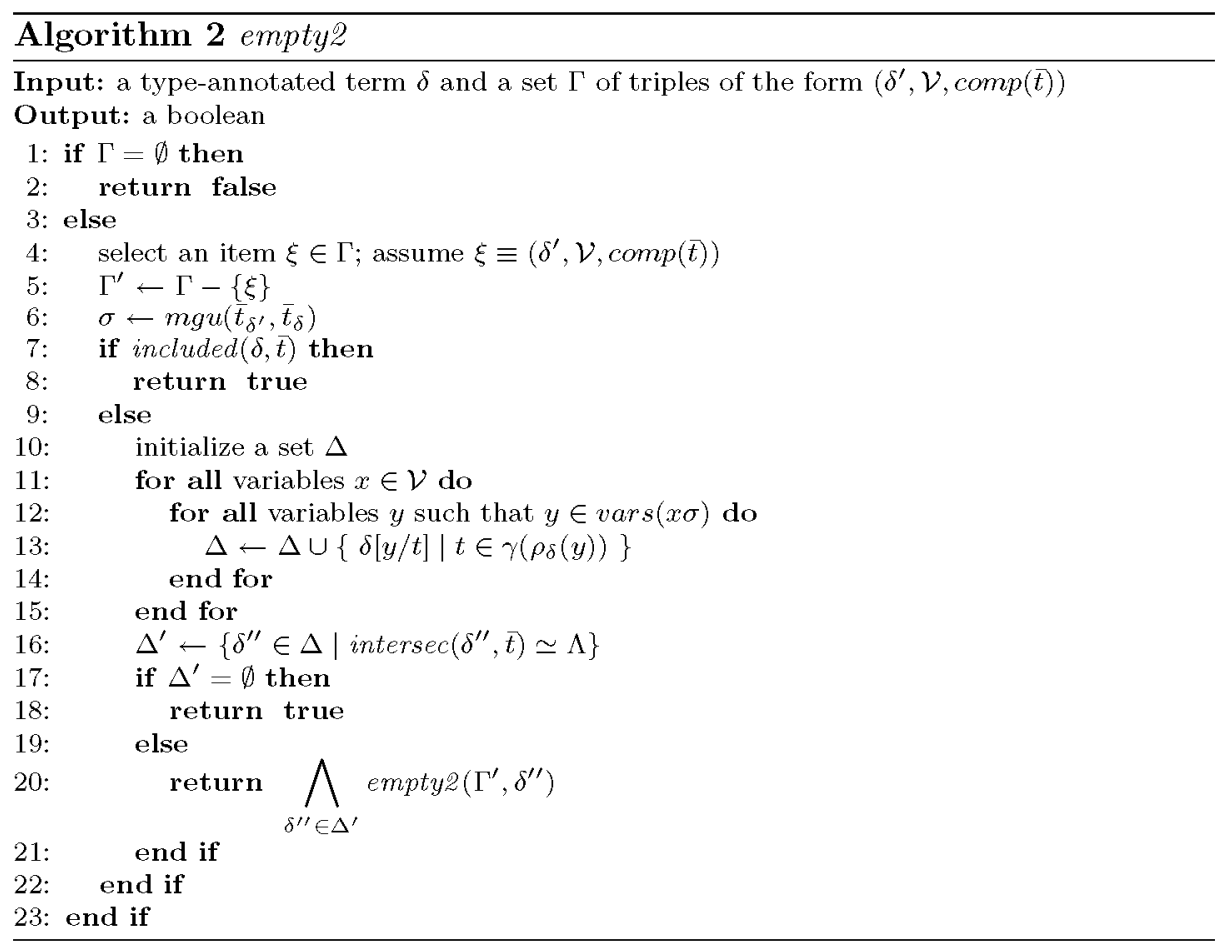

step 13 , necessarily $y \in \operatorname{var} s(\delta)$, and $\rho_{\delta}(y)$ is finite. Note also that, at step 16 , necessarily, for all $\delta^{\prime \prime} \in \Delta$ and $\delta^{\prime \prime} \notin \Delta^{\prime}$, it holds that $\delta^{\prime \prime} \sqsubseteq \bar{t}$. For this reason, $\operatorname{comp}(\bar{t})$ is removed from the recursive call at step 20 .

\section{Soundness and Completeness Results}

The function empty $(\delta, S)$ is sound and complete for tuple-distributive regular types (a detailed proof is given in ${ }^{18)}$ ). While sound, the function is not complete for regular types in general. However, our experience (as we will see in Section 5) is that it is fairly accurate in practice. Note that our applications do not require analysis algorithms to be complete (impossible in general) but rather always safe and as accurate as possible. ${ }^{11)}$

\section{Theorem 4.3}

Let $\delta$ be a type-annotated term such that all types appearing in it are tupledistributive regular types, and $\beta$ a minset with the conditions of definitions 4.13 , 4.14, and 4.15. Let also functions empty, empty1, and empty2 defined there. We have that:

1. empty, empty1, and empty2 terminate.

2. empty2 $(\Gamma, \delta)=$ true if and only if $\delta \otimes \mathcal{C} \simeq \Lambda$, where $\mathcal{C}=\{\operatorname{comp}(\bar{t}) \mid$ $\left(\delta^{\prime}, \mathcal{V}, \operatorname{comp}(\bar{t})\right) \in \Gamma$ for some $\delta^{\prime}$ and $\left.\mathcal{V}\right\}$ (i.e., $\mathcal{C}$ is the set of cobasic sets in $\Gamma$ ). 
3. $\quad$ empty $1(\mathcal{C}, \delta, \Gamma)=$ true if and only if $\delta \otimes \mathcal{C}_{1} \simeq \Lambda$, where $\mathcal{C}_{1}=\mathcal{C} \cup$ $\left\{\operatorname{comp}(\bar{t}) \mid\left(\delta_{1}, \mathcal{V}, \operatorname{comp}(\bar{t})\right) \in \Gamma\right.$ for some $\delta_{1}$ and $\left.\mathcal{V}\right\}$.

4. $\operatorname{empty}(\delta, \beta)=$ true if and only if $\delta \otimes \beta \simeq \Lambda$.

\subsection{Checking Mutual Exclusion in Linear Arithmetic}

In this section, we give an algorithm for checking whether two linear arithmetic tests $\tau_{i}(\bar{x})$ and $\tau_{j}(\bar{x})$ are exclusive w.r.t. the type assignment of int to each variable in $\bar{x}$. This amounts to determining whether $(\exists \bar{x})\left(\tau_{i}(\bar{x}) \wedge \tau_{j}(\bar{x})\right)$ is unsatisfiable. The system $\tau_{i}(\bar{x}) \wedge \tau_{j}(\bar{x})$ can be transformed into disjunctive normal form as in equation (1) below, where each of the tests $\phi_{k l}(\bar{x})$ is of the form $\phi_{k l}(\bar{x}) \equiv a_{0}+a_{1} x_{1}+\cdots+a_{p} x_{p}(?)$, with $(? \in\{=,<, \leq,>$, $\geq\}$. For this transformation, note that a test $a_{0}+a_{1} x_{1}+\cdots+a_{p} x_{p} \neq 0$ can be written in terms of two tests involving only ' $>$ ' and ' $<$ ', as in equation (2).

$$
\left(\tau_{i}(\bar{x}) \wedge \tau_{j}(\bar{x})\right)=\bigvee_{k=1}^{n} \bigwedge_{l=1}^{m} \phi_{k l}(\bar{x})
$$

(1) Disjunctive normal form

$$
\left(\sum_{i=0}^{p} a_{i} x_{i}>0\right) \vee\left(\sum_{i=0}^{p} a_{i} x_{i}<0\right)
$$

(2) Rewriting of disequalities

The resulting system, transformed to disjunctive normal form, defines a set of integer programming problems: the answer to the original mutual exclusion problem is "yes" if and only if none of these integer programming problems has a solution. Since a test can give rise to at most finitely many integer programming problems in this way, it follows that the mutual exclusion problem for linear integer tests is decidable. Since determining whether an integer programming problem is solvable is NP-complete, ${ }^{8)}$ the following complexity result is immediate:

\section{Theorem 4.4}

The mutual exclusion problem for linear arithmetic tests over the integers is co-NP-hard.

It should be noted, however, that the vast majority of arithmetic tests encountered in practice tend to be fairly simple: our experience has been that tests involving more than two variables are rare. The solvability of integer programs in the case where each inequality involves at most two variables, i.e., is of the form $a x+b y \leq c$, can be decided efficiently in polynomial time by examining the loops in a graph constructed from the inequalities. ${ }^{1)}$ The integer programming problems that arise in practice, in the context of mutual exclusion analysis, are therefore efficiently decidable.

The ideas explained in this section for linear arithmetic over integers extend directly to linear tests over the reals, which turn out to be computationally somewhat simpler.

\subsection{Checking Mutual Exclusion: Putting It All Together}

Consider a predicate $p$ defined by $n$ clauses $C_{1}, \ldots, C_{n}$, with input tests $\tau_{1}(\bar{x}), \ldots, \tau_{n}(\bar{x})$ respectively. Assume, without loss of generality, that each $\tau_{k}(\bar{x})$, 
$1 \leq k \leq n$ is a conjunction of primitive tests (note that it is always possible to obtain an equivalent sequence of clauses where disjunctions have been removed). Assume also that each $\tau_{k}(\bar{x}), 1 \leq k \leq n$, is written as $\tau_{k}^{H} \wedge \tau_{k}^{A}$, where $\tau_{k}^{H}$ and $\tau_{k}^{A}$ are a conjunction of primitive unification and arithmetic tests respectively (i.e., we write arithmetic tests after unification tests). Consider also each $\tau_{k}^{H}$ written as a minset $\beta_{k}$ (the function test2minset, given in Definition 4.6, returns the minset representation of a test).

Assume that predicate $p$ has type type $[p]$. In order to check whether $p$ is mutually exclusive (i.e., its clauses are mutually exclusive w.r.t. the type assignment $\bar{x}$ : type $[p]$ ) we need to solve the problem of determining whether any pair of tests $\tau_{i}(\bar{x})$ and $\tau_{j}(\bar{x}), 1 \leq i, j \leq n, i \neq j$, is exclusive w.r.t. $\bar{x}$ : type $[p]$.

Before describing a sufficient condition for ensuring that these tests are exclusive, we define some instrumental elements. Let $\beta_{i j}$ be the minset intersection of $\beta_{i}$ and $\beta_{j}$. Let $\theta_{i}$ (resp. $\theta_{j}$ ), be the most general unifier of the tuple of terms of $\beta_{i j}$ and $\beta_{i}$ (resp. $\beta_{j}$ ). That is, if $\beta_{i} \equiv \bar{t}_{i} \otimes \mathcal{C}_{i}, \beta_{j} \equiv \bar{t}_{j} \otimes \mathcal{C}_{j}$, and $\beta_{i j} \equiv \bar{t}_{i j} \otimes \mathcal{C}_{i j}$, then $\theta_{i}=m g u\left(\bar{t}_{i}, \bar{t}_{i j}\right), \bar{t}_{i j} \equiv \bar{t}_{i} \theta_{i}, \theta_{j}=m g u\left(\bar{t}_{j}, \bar{t}_{i j}\right), \bar{t}_{i j} \equiv \bar{t}_{j} \theta_{j}$ (note that there exists a substitution $\mu_{i j}$, such that $\mu_{i j}=m g u\left(\bar{t}_{i}, \bar{t}_{j}\right)$ ). Let $\rho$ be the type assignment $\bar{x}:$ type $[p]$ but written as a type-annotated term $\delta$. We have that the tests $\tau_{i}(\bar{x})$ and $\tau_{j}(\bar{x})$, are exclusive w.r.t. $\rho$ if:

1. $\delta \otimes \beta_{i} \otimes \beta_{j} \simeq \Lambda$ (which can be checked as explained in Section 4.1), or

2. $\delta \otimes \beta_{i} \otimes \beta_{j} \neq \Lambda$ and $\tau_{i}^{A} \theta_{i} \wedge \tau_{j}^{A} \theta_{j}$ is unsatisfiable (which can be checked as explained in Section 4.2 ).

\section{Example 4.6}

Reconsider Example 4.1 with predicate part/4 from the quicksort program of Fig. 1. We had reduced the mutual exclusion problem to two subproblems: a) checking that the tests $\mathrm{L}=[]$ and $\mathrm{L}=[\mathrm{E} \mid \mathrm{R}]$ are exclusive w.r.t. type assignment $\rho$, and $\mathrm{b}$ ) checking that the tests $\mathrm{E}\langle\mathrm{C}$ and $\mathrm{E}\rangle=\mathrm{C}$ are exclusive w.r.t. $\rho$. In this case, we have that $\delta$ is $((\mathrm{L}, \mathrm{C}),(\mathrm{L}$ : intlist, $\mathrm{C}$ : int $))$. Also, $\beta_{1} \equiv([], C)$, $\beta_{2} \equiv([\mathrm{E} \mid \mathrm{R}], \mathrm{C})$, and $\beta_{3} \equiv([\mathrm{E} \mid \mathrm{R}], \mathrm{C})$. We now have that part/4 is mutually exclusive because: $\delta \otimes \beta_{i} \otimes \beta_{j} \simeq \Lambda$, for $i=1$ and $j \in\{2,3\}$, and (although $\delta \otimes \beta_{2} \otimes \beta_{3} \not \Lambda$ ) also $\mathrm{E}<\mathrm{C} \wedge \mathrm{E} \geq \mathrm{C}$ is unsatisfiable (note that $\beta_{2,3} \equiv([\mathrm{E} \mid \mathrm{R}], \mathrm{C}$ ), and $\theta_{2}$ and $\theta_{3}$ are the identity).

\subsection{Checking Mutual Exclusion: Dealing with the Cut}

The presence of a pruning operator (cut) in program clauses can help the detection of mutual exclusion. In order to take the cut into account, we simply redefine the concept of mutually exclusive clauses in Definition 2.4 as:

\section{Definition 4.16 (mutual exclusion in the presence of cut)}

Let $C_{1}, \ldots, C_{n}, n>0$, be a sequence of clauses, with input tests $\tau_{1}, \ldots, \tau_{n}$ respectively. Let $\rho$ be a type assignment. We say that $C_{1}, \ldots, C_{n}$ is mutually exclusive w.r.t. $\rho$ if either, $n=1$, or, for every pair of clauses $C_{i}$ and $C_{j}$, $1 \leq i, j \leq n, i \neq j$ : $C_{i}$ has a cut and $i<j$, or $C_{j}$ has a cut and $j<i$, or 
$\tau_{i}(\bar{x})$ and $\tau_{j}(\bar{x})$ are exclusive w.r.t. $\rho$.

We also have to take into account that the pruning operator introduces implicit tests. Consider a predicate $p$ defined by a sequence of $n$ clauses $C_{1}, \ldots, C_{n}$, with input tests $\tau_{1}(\bar{x}), \ldots, \tau_{n}(\bar{x})$ respectively. Let $I$ be the set of indexes $k$ of clauses $C_{k}$ which have a cut and are before the clause $C_{i}$ (i.e., $k<i$ ). Let $\tau_{k}^{b}$ be the test (conjunction of tests) that is before the cut in clause $C_{k}$ (i.e., $\tau_{k} \equiv \tau_{k}^{b} \wedge \tau_{k}^{a}$, where $\tau_{k}^{a}$ is the test that is after the cut in clause $C_{k}$ ). Now, instead of considering the test $\tau_{i}$, for $1 \leq i \leq n$, in Definition 4.16 , we take the test $\tau_{i}^{c}$ defined as follows:

$$
\tau_{i}^{c}=\left(\bigwedge_{k \in I} \neg \tau_{k}^{b}\right) \wedge \tau_{i}
$$

\section{Example 4.7}

Consider predicate abs $/ 2$ mentioned in page 183 . Usually, this predicate is defined with a cut in the first clause and no check in the second. In this case, the test for the second clause will be $\neg \mathrm{X} \geq 0$.

Note that the introduction of negation in the tests $\tau_{i}^{c}$ is not a problem, since it is always possible to reduce the problem of determining whether a pair of tests $\tau_{i}^{c}$ and $\tau_{j}^{c}$ are exclusive w.r.t. a given type assignment to one or more exclusion subproblems where the pair of tests involved in each subproblem are conjunctions of primitive tests (transforming tests to disjunctive normal form).

\section{$\S 5$ A Prototype Implementation}

In order to evaluate the effectiveness and efficiency of our approach to determinacy analysis, we have constructed a prototype which performs such analysis in an automatic way. The system takes Prolog programs as input, ${ }^{* 3}$ which include a module definition in the standard way. In addition, the types and modes of the arguments of exported predicates are either given or obtained from other modules during modular type and mode analysis (including the intervening type definitions). The system uses the CiaoPP PLAI analyzer to derive mode information, using, for the reported experiments, the Sharing+Freeness domain, ${ }^{21)}$ and the eterms domain to derive the types of predicates. ${ }^{25)}$ The resulting typeand mode-annotated programs are then analyzed using the algorithms presented for Herbrand and linear arithmetic tests.

Herbrand mutual exclusion is checked by a naive direct implementation of the analyses presented. Testing of mutual exclusion for linear arithmetic tests is implemented directly using the Omega test. ${ }^{22)}$ This test determines whether there is an integer solution to an arbitrary set of linear equalities and inequalities, referred to as a problem.

\footnotetext{
*3 In fact, the input language currently supported includes also a number of extensions such as functions or feature terms - which are translated by the first (expansion) passes of the Ciao compiler to clauses, possibly with cut.
} 
Table 1 Accuracy and Efficiency of the Determinacy Analysis (times in $\mathrm{mS}$ ).

\begin{tabular}{|l|r|r|r|r|r|r|r|}
\hline Program & $\mathbf{N}$ & $\mathbf{D}(\mathbf{\%})$ & $\mathbf{M}(\boldsymbol{\%})$ & $\mathbf{C}$ & $\mathbf{T}_{D}$ & $\mathbf{T}_{M}$ & $\mathbf{T}_{T}$ \\
\hline \hline Hanoi & 2 & $2(100)$ & $2(100)$ & N/A & 48 & 55 & 103 \\
\hline Fib & 1 & $1(100)$ & $1(100)$ & N/A & 16 & 21 & 37 \\
\hline Mmatrix & 3 & $3(100)$ & $3(100)$ & N/A & 24 & 39 & 63 \\
\hline Tak & 1 & $1(100)$ & $1(100)$ & N/A & 24 & 23 & 47 \\
\hline Subs & 1 & $1(100)$ & $1(100)$ & N/A & 12 & 16 & 28 \\
\hline Reverse & 2 & $2(100)$ & $2(100)$ & N/A & 21 & 20 & 41 \\
\hline \hline Qsort & 3 & $3(100)$ & $3(100)$ & $3(100)$ & 40 & 34 & 74 \\
\hline Qsort2 & 5 & $5(100)$ & $5(100)$ & $5(100)$ & 64 & 43 & 107 \\
\hline Queens & 6 & $3(50)$ & $5(83)$ & $2(33)$ & 65 & 36 & 101 \\
\hline Gabriel & 20 & $6(30)$ & $11(55)$ & $4(20)$ & 308 & 241 & 549 \\
\hline Kalah & 44 & $40(91)$ & $42(95)$ & $40(91)$ & 952 & 2432 & 3384 \\
\hline Plan & 16 & $8(50)$ & $12(75)$ & $3(19)$ & 402 & 811 & 1213 \\
\hline Credit & 25 & $18(72)$ & $21(84)$ & $16(64)$ & 1032 & 321 & 1353 \\
\hline Pg & 10 & $6(60)$ & $9(90)$ & $6(60)$ & 372 & 177 & 549 \\
\hline \hline Mean & - & $71 \%$ & $85 \%$ & $61 \%$ & $24(/ \mathrm{p})$ & $31(/ \mathrm{p})$ & $55(/ \mathrm{p})$ \\
\hline \hline
\end{tabular}

We have tested the prototype first on a number of simple standard benchmarks, and then on more complex ones. The latter are taken from those used in the cardinality analysis of Braem et al. ${ }^{2)}$ which, as mentioned in Section 1, is the closest related previous work that we are aware of. In the case of Kalah, we have inserted the missing cuts as is also done in ${ }^{2)}$, to make the comparison meaningful. Some relevant results of these tests are presented in Table 1. Program lists the program names, $\mathbf{N}$ the number of predicates in the program, $\mathbf{D}$ the number of them detected by the analysis as deterministic, $\mathbf{M}$ the number of predicates whose tests are mutually exclusive, $\mathbf{C}$ the number of deterministic predicates detected in ${ }^{2)}, \mathbf{T}_{D}$ the time required by the determinacy analysis (Ciao/CiaoPP version 1.13, rev 10683, on an Intel Pentium M 1.86GHz, $1 \mathrm{~Gb}$ of RAM memory, running Ubuntu Linux 8.04, and averaging several runs, eliminating the best and worst values), $\mathbf{T}_{M}$ the time required to derive the modes and types, and $\mathbf{T}_{T}$ the total analysis time (all times are in milliseconds). Averages (per predicate in the case of analysis time) are also provided in the last row of the table.

The results are quite encouraging, showing that the developed analysis is fairly accurate. The analysis is more powerful in some cases than the cardinality analysis, ${ }^{2)}$ and at least as accurate in the others. It is pointed out in ${ }^{2)}$ that determinacy information can be improved by using a more sophisticated type domain. This is also applicable to our analysis, and the types inferred by our system are similar to those used in ${ }^{2)}$. The determinacy analysis times are also encouraging, despite the currently relatively naive implementation of the system (for example, the call to the Omega test is done by calling an external process). The overall analysis times are also reasonable, even when including the type and mode analysis times, which are in any case very useful in other parts of the compilation process. 


\section{$\S 6$ Conclusion}

We have proposed an analysis for detecting procedures and goals that are deterministic (i.e., that produce at most one solution at most once), or predicates whose clause tests are mutually exclusive, even if they are not deterministic (because they call other predicates which are nondeterministic). Our approach has advantages w.r.t. previous approaches in that it provides an algorithm for detecting mutual exclusion and it handles disunification tests on the Herbrand domain and arithmetic tests.

We have implemented the proposed analysis and integrated it into the CiaoPP system, which also infers automatically the mode and type information that the proposed analysis takes as input. The results of the experiments performed on this implementation show that the analysis is fairly accurate and efficient, providing more accurate or similar results, regarding accuracy, than previous proposals, while offering substantially higher automation, since typically no information is needed from the user.

\section{Acknowledgements}

This work has been supported in part by the Information Society Technologies program of the European Commission, FP6 FET IST-15905 MOBIUS, IST-215483 SCUBE, and 06042-ESPASS, Ministry of Science projects TIN-200805624 DOVES, TIN2005-09207-C03 MERIT-COMVERS, Ministry of Industry project FIT-340005-2007-14, and CAM project S-0505/TIC/0407 PROMESAS.

\section{References}

1) Aspvall, B. and Shiloach, Y., "A polynomial time algorithm for solving systems of linear inequalities with two variables per inequality," in Proc. 20th ACM Symposium on Foundations of Computer Science, pp. 205-217, October 1979.

2) Braem, C., Le Charlier, B., Modart, S. and Van Hentenryck, P., "Cardinality analysis of prolog," in Proc. International Symposium on Logic Programming, pp. 457-471, Ithaca, NY, MIT Press, November 1994.

3) Bueno, F., Deransart, P., Drabent, W., Ferrand, G., Hermenegildo, M., Maluszynski, J. and Puebla, G., "On the Role of Semantic Approximations in Validation and Diagnosis of Constraint Logic Programs," in Proc. of the 3rd. Int'l Workshop on Automated Debugging-AADEBUG' 97, pp. 155-170, Linköping, Sweden, U. of Linköping Press, May 1997.

4) Dart, P.W. and Zobel, J., "A Regular Type Language for Logic Programs," in Types in Logic Programming, pp. 157-187, MIT Press, 1992.

5) Dawson, S., Ramakrishnan, C. R., Ramakrishnan, I. V. and Sekar, R. C., "Extracting Determinacy in Logic Programs," in 1993 International Conference on Logic Programming, pp. 424-438, MIT Press, June 1993.

6) Debray, S. K. and Warren, D. S., "Functional computations in logic programs," ACM Transactions on Programming Languages and Systems, 11, 3, pp. 451-481, 1989. 
7) Demoen, B., García de la Banda, M., Harvey, W., Marriott, K. and Stuckey, P., "An Overview of HAL," in 5th International Conference on Principles and Practice of Constraint Programming (CP' 99), vol. 1713 of LNCS (Jaffar, J. ed.), pp. 174-188, Springer-Verlag, October 1999.

8) Garey, M. R. and Johnson, D. S., Computers and Intractability: A Guide to the Theory of NP-Completeness, W.H. Freeman, New York, 1979.

9) Giacobazzi, R. and Ricci, L., "Detecting Determinate Computations by Bottomup Abstract Interpretation," in 4th European Symposium on Programming (ESOP'92), vol. 582 of LNCS (Krieg-Brückner, B. ed.), pp. 167-181, SpringerVerlag, February 1992.

10) Henderson, F., Somogyi, Z. and Conway, T., "Determinism Analysis in the Mercury Compiler," in Proc. of the 9th Australian Computer Science Conference, vol. 18 of Australian Computer Science Communications (Kotagiri, R. ed.), pp. 337-346, RMIT, The University of Melbourne, January 1996.

11) Hermenegildo, M., Puebla, G., Bueno, F. and López-García, P., "Integrated Program Debugging, Verification, and Optimization Using Abstract Interpretation (and The Ciao System Preprocessor)," Science of Computer Programming, 58, 1-2, pp. 115-140, October 2005.

12) Hill, P. M. and King, A., "Determinacy and determinacy analysis," Journal of Programming Languages, 5, 1, pp. 135-171, December 1997.

13) Janssens, G. and Bruynooghe, M., "Deriving Descriptions of Possible Values of Program Variables by means of Abstract Interpretation," Journal of Logic Programming, 13, 2-3, pp. 205-258, July 1992.

14) King, A., Lu, L. and Genaim, S., "Detecting Determinacy in Prolog Programs," in Logic Programming, 22nd International Conference, ICLP 2006, Seattle, WA, USA, August 17-20, 2006, Proc., vol. 4079 of LNCS (Etalle, S. and Truszczynski, M. eds.), pp. 132-147, Springer, 2006.

15) Kunen, K., "Answer Sets and Negation as Failure," in Proc. of the Fourth International Conference on Logic Programming, pp. 219-228, MIT Press, Melbourne, May 1987.

16) Lassez, J.-L., Maher, M. and Marriott, K., "Unification Revisited," in Foundations of Deductive Databases and Logic Programming (Minker, J. ed.), pp. 587-626, Morgan Kaufmann, 1988.

17) López-García, P., Bueno, F. and Hermenegildo, M., "Determinacy Analysis for Logic Programs Using Mode and Type Information," in Proc. of the 14th International Symposium on Logic-based Program Synthesis and Transformation (LOPSTR'04), vol. 3573 of LNCS, pp. 19-35, Springer-Verlag, August 2005.

18) López-García, P., Bueno, F. and Hermenegildo, M., "Inferring Determinacy and Mutual Exclusion in Logic Programs Using Mode and Type Analysis," Technical Report CLIP2/2009.0, Technical University of Madrid (UPM), School of Computer Science, UPM, February 2009.

19) Lu, L. and King, A., "Determinacy Inference for Logic Programs," in Programming Languages and Systems, 14 th European Symposium on Programming (ESOP 2005), vol. 3444 of LNCS (Sagiv, M. ed.), pp. 108-123, Springer, 2005.

20) Æ. Mogensen, T., "A Semantics-Based Determinacy Analysis for Prolog with Cut," in Perspectives of System Informatics, Second International Andrei Ershov Memorial Conference, vol. 1181 of LNCS, pp. 374-385, Springer, 1996. 
21) Muthukumar, K. and Hermenegildo M., "Combined Determination of Sharing and Freeness of Program Variables through Abstract Interpretation," in 1991 International Conference on Logic Programming, pp. 49-63, MIT Press, June 1991.

22) Pugh, W., "A Practical Algorithm for Exact Array Dependence Analysis," Communications of the ACM, 35, 8, pp. 102-114, August 1992.

23) Sahlin, D., "Determinacy Analysis for Full Prolog," in Proc. of the 1991 ACM SIGPLAN Symposium on Partial Evaluation and Semantics-based Program Manipulation (PEPM'91), pp. 23-30, ACM Press, 1991.

24) Somogyi, Z., Henderson, F. and Conway, T., "The Execution Algorithm of Mercury: an Efficient Purely Declarative Logic Programming Language," Journal of Logic Programming, 29, 1-3, pp. 17-64, October 1996.

25) Vaucheret, C. and Bueno, F., "More Precise yet Efficient Type Inference for Logic Programs," in International Static Analysis Symposium, vol. 2477 of LNCS, pp. 102-116, Springer-Verlag, September 2002. 